\title{
Macroscopic Response of Particle-Reinforced Elastomers Subjected to Prescribed Torques or Rotations on the Particles
}

\author{
Morteza H. Siboni ${ }^{\mathrm{a}}$, Pedro Ponte Castañeda ${ }^{\mathrm{a}, *}$ \\ ${ }^{a}$ Department of Mechanical Engineering and Applied Mechanics \\ University of Pennsylvania, Philadelphia, PA
}

\begin{abstract}
Particle-reinforced rubbers are composite materials consisting of randomly distributed, stiff fibers/particles in a soft elastomeric material. Since the particles are stiff compared to the embedding rubber, their deformation can be ignored for all practical purposes. However, due to the softness of the rubber, they can undergo rigid body translations and rotations. Constitutive models accounting for the effect of such particle motions on the macroscopic response under prescribed deformations on the boundary have been developed recently. But, in some applications (e.g., magneto-active elastomers), the particles may experience additional torques as a consequence of an externally applied (magnetic) field, which, in turn, can affect the overall rotation of the particles in the rubber, and therefore also the macroscopic response of the composite. This paper is concerned with the development of constitutive models for particle-reinforced elastomers, which are designed to account for externally applied torques on the internally distributed particles, in addition to the externally applied deformation on the boundary of the composite. For this purpose, we propose a new variational framework involving suitably prescribed eigenstresses on the particles. For simplicity, the framework is applied to an elastomer reinforced by aligned, rigid, cylindrical fibers of elliptical cross section, which can undergo finite rotations in the context of a finite-deformation, plane strain problem for the composite. In particular, expressions are derived for the average in-plane rotation of the fibers as a function of the torques that are applied on them, both under vanishing and prescribed strain on the boundary. The results of this work will make possible the development of improved constitutive models for magneto-active elastomers, and other types of smart composite materials that are susceptible to externally applied torques.
\end{abstract}

Keywords: Finite Deformations, Rotations, Torques, Effective Response

\section{Introduction}

It has recently been proposed that the performance of magneto-active elastomers (MAEs) may be enhanced by the use of non-spherical particles (Ponte Castañeda and Galipeau, 2011; Galipeau and Ponte Castañeda, 2013). This is because the application of magnetic fields induces mechanical torques on nonspherical particles, which can be harnessed to produce large field-activated strains in MAEs consisting of

\footnotetext{
${ }^{*}$ Corresponding author

Email addresses: mortezah@nyu.edu (Morteza H. Siboni), ponte@seas.upenn.edu (Pedro Ponte Castañeda)
} 
aligned particles that are distributed randomly in an elastomer matrix. Indeed, the use of spherical particles induces only dipolar forces, which require close proximity of the particles - and therefore relatively large particle concentrations - to be effective in generating large forces. However, large particle concentrations also tend to increase the macroscopic stiffness of the composite, leading to the net result that the fieldactivated strains are relatively small in MAEs with spherical particles. On the other hand, non-spherical particles interact directly with the applied magnetic field and do not require the presence of other particles to experience torques. For this reason, they can be more effective in generating large field-activated strains, since large particle concentrations are not required. Completely analogous effects are present in dielectric elastomer composites (DECs) consisting of stiff dielectric particles in soft elastomer matrices, where the torques are generated by electrical fields instead of magnetic fields (Ponte Castañeda and Siboni, 2012; Siboni and Ponte Castañeda, 2014). Ignoring for the time being the source of the torques on the particles, this raises an interesting question in the theory of composite materials that seems to have been largely neglected thus far in the literature: How does the presence of externally produced torques on particles affect the macroscopic response of composite materials? In this work, we attempt to provide an answer to this question by generating estimates for the macroscopic response of particle-reinforced elastomers subjected to a combination of externally applied deformation on the outer boundary of the specimen and prescribed torques or rotations on the particles inside the composite. For simplicity, we will focus our attention here on the special case of fiber-reinforced elastomers with aligned fibers of elliptical cross section, but we consider finite strains and rotations. However, we should emphasize that the procedure is quite general and can be used for more general ellipsoidal particle shapes (e.g., Avazmohammadi and Ponte Castañeda, 2014a,b). As will be discussed further below in more detail, the solutions developed in this work for the effect of torques on particle-reinforced systems will allow the generation of improved estimates for MAEs and DECs in future work.

Although, to the best of the authors' knowledge, the above-mentioned problem has yet not been considered in the literature - even in the context of linear elasticity — it should be mentioned that a generalization of the single rigid inclusion problem (Eshelby, 1957) to account for additional particle rotations has been investigated by Walpole (1991). In addition, an application of this result to account for magnetic torques on a single ellipsoidal rigid particle embedded in an infinite linear-elastic medium has been considered recently by Siboni and Ponte Castañeda (2012). Finally, there are classical solutions (Rivlin, 1949; Kanner and Horgan, 2008) in the context of finite elasticity for cylindrical shells undergoing azimuthal shear that can be reinterpreted as solutions for the finite twist of single fibers of circular cross section in an elastomeric matrix.

In this paper we investigate the effect of externally applied torques or prescribed rotations on particles in composite materials, in the finite-deformation regime. This is, in general, a difficult task due to the material and geometric nonlinearities associated with finite deformations, and for this reason we have to resort to approximate solutions. In particular, we will make use of the second-order linear comparison homogenization method of Ponte Castañeda (2002). This will allow us to obtain estimates for the effective energy function 
of composites with prescribed rotations (or torques) on the particles, as well as corresponding estimates for the relation between the resulting rotations and the externally applied torques. The resulting constitutive model accounts for simultaneously prescribed deformations on the boundary of the specimen, and thus reduces to the results of Lopez-Pamies and Ponte Castañeda (2006b) for the special case when the externally applied torques on the fibers vanish, so that the particles rotate freely as a consequence of the surrounding deformation in the elastomeric matrix phase of the composite.

The rest of this paper is organized as follows. In section 2 , we provide a brief overview of the homogenization problem for hyperelastic composites in the large deformation regime. Section 3 is concerned with the generalization of the homogenization procedure for cases where uniform eigenstresses are applied externally in each phase of the composite. This will be accomplished by making use of the fact that the effects of the applied eigenstresses can be accounted for by the addition of suitably chosen linear terms (in the deformation) to the phase energy functions. Section 4 is concerned with the second-order homogenization method. In particular, we consider the application of the generalized-secant, second-order method of Lopez-Pamies and Ponte Castañeda (2006a) for the modified variational problem with eigenstress, introduced in section 3. Explicit expressions will be provided for both the effective energy function and for the average deformation of the phases in particulate composites. In section 5, we focus our attention on two-phase composite with zero eigenstress in the matrix phase and non-zero eigenstress in the inclusion phase. We also provide specializations for two-phase composites consisting of rigid inclusions. Section 6 is concerned with the derivation of an explicit expression for the effective energy and the associated microstructure evolution for composites reinforced by long rigid fibers under $2 \mathrm{D}$ plane-strain conditions. In particular, explicit expressions are derived for the effective energy and average in-plane rotation of the fibers as a function of the prescribed eigenstress, which can then be related to the externally applied body couples (see Appendix A). In section

7 , we first compare the torque-rotation relation obtained in this paper with previously available results for single fibers of circular cross section. We then explore the effect of different microstructural variables, as well as the macroscopic deformation, on the torque-rotation response of fibers. We also investigate the effect of externally applied torques on the macroscopic constitutive response of the composite as determined by the relation between the effective Cauchy stress and the macroscopic deformation. Finally, in section 8 we conclude the paper by discussing our findings and possible future directions.

\section{Effective behavior}

We consider a composite specimen, occupying the volume $\Omega_{0}$ in the reference configuration, that consists of $N$ different phases with uniform but nonlinear stored-energy functions $W^{(r)}(\boldsymbol{F})$. As usual, $\boldsymbol{F}:=$ $\operatorname{Grad} \boldsymbol{\chi}(\mathbf{X})$ denotes the deformation gradient tensor of the map $\mathbf{x}=\boldsymbol{\chi}(\mathbf{X})$ between the material points in the reference $(\mathbf{X})$ and deformed $(\mathbf{x})$ configurations. When the affine boundary condition,

$$
\mathbf{x}=\overline{\boldsymbol{F}} \mathbf{X} \quad \text { for } \quad \mathbf{X} \text { on } \partial \Omega_{0}
$$


is applied on the boundary of the sample, and under the assumptions of the separation of length scales hypothesis, the effective constitutive response of the above-mentioned composite may be characterized by the effective potential $\tilde{W}(\overline{\boldsymbol{F}})$, as obtained from the "standard" variational problem

$$
\tilde{W}(\overline{\boldsymbol{F}})=\min _{\boldsymbol{F} \in \mathcal{K}(\overline{\boldsymbol{F}})}\langle W(\mathbf{X}, \boldsymbol{F})\rangle_{0}=\min _{\boldsymbol{F} \in \mathcal{K}(\overline{\boldsymbol{F}})} \sum_{r=1}^{N} c_{0}^{(r)}\left\langle W^{(r)}(\boldsymbol{F})\right\rangle_{0}^{(r)} .
$$

Here $\langle\cdot\rangle_{0}$ and $\langle\cdot\rangle_{0}^{(r)}$ are the volume average operations over the volumes $\Omega_{0}$ and $\Omega_{0}^{(r)}$, respectively, and $W(\mathbf{X}, \boldsymbol{F})$ is the local potential of the composite that can be written in terms of the phase potentials $W^{(r)}(\boldsymbol{F})$ and phase characteristic functions $\Theta^{(r)}(\mathbf{X})$ as follows

$$
W(\mathbf{X}, \boldsymbol{F})=\sum_{r=1}^{N} \Theta^{(r)}(\mathbf{X}) W^{(r)}(\boldsymbol{F}), \quad \text { where } \quad \Theta^{(r)}(\mathbf{X})=1 \text { for } \mathbf{X} \in \Omega_{0}^{(r)}, \text { and } 0 \text { otherwise. }
$$

In addition, $\mathcal{K}(\overline{\boldsymbol{F}})$ denotes the set of admissible deformation gradients $\boldsymbol{F}$ satisfying the affine boundary conditions (1), i.e.,

$$
\mathcal{K}(\overline{\boldsymbol{F}})=\left\{\boldsymbol{F} \mid \exists \mathbf{x}=\chi(\mathbf{X}), \text { such that } \boldsymbol{F}=\operatorname{Grad} \boldsymbol{\chi}, \text { and } \mathbf{x}=\overline{\boldsymbol{F}} \mathbf{X} \text { on } \partial \Omega_{0}\right\}
$$

Note that $\langle\boldsymbol{F}\rangle_{0}=\overline{\boldsymbol{F}}$ and that $\tilde{W}(\overline{\boldsymbol{F}})$ corresponds to the average elastic energy stored in the composite when subjected to an affine boundary condition of the form (1). The global, or macroscopic, constitutive relation for the composite is then obtained (Hill, 1972) via

$$
\overline{\boldsymbol{S}}=\frac{\partial \tilde{W}}{\partial \overline{\boldsymbol{F}}}
$$

where $\overline{\boldsymbol{S}}:=\langle\boldsymbol{S}\rangle_{0}$ denotes the average (or macroscopic) Piola-Kirchhoff stress in the composite.

It is often of interest to have a measure for the evolution of the microstructure (especially for composites undergoing large deformations), as determined by the averages of the deformation gradient in the phases of the nonlinear composite. Thus, the average deformation gradient in phase $s$ of the nonlinear composite may be obtained (see Idiart and Ponte Castañeda, 2007, for more details) by means of the identities

$$
\overline{\boldsymbol{F}}^{(s)}:=\langle\boldsymbol{F}\rangle_{0}^{(s)}=\left.\frac{1}{c_{0}^{(s)}} \frac{\partial \tilde{W}_{\boldsymbol{T}}}{\partial \boldsymbol{T}^{(s)}}\right|_{\boldsymbol{T}^{(s)}=0}, \quad s=1, \ldots, N,
$$

where $\tilde{W}_{\boldsymbol{T}}(\boldsymbol{F})$, denotes the effective energy of a fictitious composite with the same microstructure as the original composite, but with the following "perturbed" local energy

$$
W_{\boldsymbol{T}}(\mathbf{X}, \boldsymbol{F}):=\sum_{r=1}^{N} \Theta^{(r)}(\mathbf{X}) W^{(r)}(\boldsymbol{F})+\Theta^{(s)}(\mathbf{X}) \boldsymbol{T}^{(s)} \cdot \boldsymbol{F} .
$$

Note that the constant second-order tensor $\boldsymbol{T}^{(s)}$ on the right side of (7) can be thought of as a uniform eigenstress (or thermal stress) applied in phase $s$ of the nonlinear composite. 


\section{Composites under the application of uniform-per-phase eigenstresses}

In this section, we address the homogenization problem for composites under the application of external eigenstresses. (This problem will be related further below to the problem of prescribed torques and rotations on the fibers in a fiber-reinforced elastomer.) Thus, we consider an $N$-phase composite, whose microstructure is described by the characteristic functions $\Theta^{(r)}(\mathbf{X})$ for $r=1, \ldots, N$, under the application of a local eigenstress of the form

$$
\boldsymbol{M}(\mathbf{X})=\sum_{r=1}^{N} \Theta^{(r)}(\mathbf{X}) \boldsymbol{M}^{(r)},
$$

where $\boldsymbol{M}^{(r)}$ denotes the externally applied eigenstress in phase $r$ of the composite. (As shown in Appendix A, the second-order tensors $\boldsymbol{M}^{(r)}$ can be used to characterize externally applied body couples.) Then the effective energy of the composite under the application of the eigenstresses $\boldsymbol{M}^{(s)}(s=1, \ldots, N)$, denoted here by $\tilde{W}_{\boldsymbol{M}}\left(\overline{\boldsymbol{F}} ; \boldsymbol{M}^{(s)}\right)$, may be obtained via the "modified" variational problem

$$
\tilde{W}_{\boldsymbol{M}}\left(\overline{\boldsymbol{F}} ; \boldsymbol{M}^{(s)}\right)=\min _{\boldsymbol{F} \in \mathcal{K}(\overline{\boldsymbol{F}})}\left\langle W_{\boldsymbol{M}}(\mathbf{X}, \boldsymbol{F})\right\rangle_{0}
$$

where the "modified" local energy $W_{\boldsymbol{M}}(\mathbf{X}, \boldsymbol{F})$ is defined as

$$
W_{\boldsymbol{M}}(\mathbf{X}, \boldsymbol{F}):=\sum_{r=1}^{N} \Theta^{(r)}(\mathbf{X})\left[W^{(r)}(\boldsymbol{F})+\boldsymbol{M}^{(r)} \cdot \boldsymbol{F}\right] .
$$

The evolution of the microstructure for the above composite may be determined by means of the prescriptions of section 2. Thus, it is easily shown that the averages of the deformation gradient in the phases of the composite under the application of the uniform eigenstresses $\boldsymbol{M}^{(s)}$ may be obtained in terms of the derivatives of the effective energy $\tilde{W}_{\boldsymbol{M}}\left(\boldsymbol{F} ; \boldsymbol{M}^{(s)}\right)$ with respect to the second-order tensors $\boldsymbol{M}^{(s)}$, i.e.,

$$
\overline{\boldsymbol{F}}^{(s)}=\frac{1}{c_{0}^{(s)}} \frac{\partial}{\partial \boldsymbol{M}^{(s)}} \tilde{W}_{\boldsymbol{M}}\left(\overline{\boldsymbol{F}} ; \boldsymbol{M}^{(r)}\right), \quad \text { for } \quad s=1, \ldots, N .
$$

Note that the phase averages $\overline{\boldsymbol{F}}^{(s)}$ satisfy the condition $\sum_{r=1}^{N} c_{0}^{(r)} \overline{\boldsymbol{F}}^{(r)}=\overline{\boldsymbol{F}}$.

The expressions (11) can then be used to establish Legendre "duality" relations between the prescribed eigenstresses $\boldsymbol{M}^{(s)}$ and the average deformation gradients $\overline{\boldsymbol{F}}^{(s)}$. For this purpose, we define the effective energy of the $N$-phase composite with prescribed average deformation gradients $\boldsymbol{F}^{(s)}$ via the Legendre transformation

$$
\tilde{W}_{\boldsymbol{F}}\left(\overline{\boldsymbol{F}} ; \boldsymbol{F}^{(s)}\right)=\operatorname{stat}_{\boldsymbol{M}^{(s)}}\left[\sum_{r=1}^{N} c_{0}^{(r)} \boldsymbol{M}^{(r)} \cdot \boldsymbol{F}^{(r)}-\tilde{W}_{\boldsymbol{M}}\left(\overline{\boldsymbol{F}} ; \boldsymbol{M}^{(s)}\right)\right],
$$

where the stat(ionary) operation corresponds to taking the derivative of the terms in the brackets with respect to the variables $\boldsymbol{M}^{(s)}(s=1, \ldots, N)$, solving for these variables in terms of the corresponding variables $\boldsymbol{F}^{(s)}$, and plugging the results back in the expression. Thus, the effective energy $\tilde{W}_{\boldsymbol{F}}\left(\overline{\boldsymbol{F}} ; \boldsymbol{F}^{(s)}\right)$ can be thought of as the Legendre dual of the energy $\tilde{W}_{\boldsymbol{M}}\left(\overline{\boldsymbol{F}} ; \boldsymbol{M}^{(s)}\right)$ (with respect to the variables $\boldsymbol{M}^{(s)}$ ). As expected, the stationary conditions associated with (12) are precisely the same as those given by (11), which establishes the desired duality relations between the variables $\boldsymbol{M}^{(s)}$ and $\boldsymbol{F}^{(s)}$. 
It is emphasized at this point that the effective energy of the composite with prescribed macroscopic deformations for the phases, $\tilde{W}_{\boldsymbol{F}}\left(\overline{\boldsymbol{F}} ; \boldsymbol{F}^{(s)}\right)$, can also be obtained, in a more direct fashion, by means of the "constrained" variational problem

$$
\tilde{W}_{\boldsymbol{F}}\left(\overline{\boldsymbol{F}} ; \boldsymbol{F}^{(s)}\right)=-\min _{\boldsymbol{F} \in \mathcal{K}_{C}(\overline{\boldsymbol{F}})}\langle W(\mathbf{X}, \boldsymbol{F})\rangle_{0}
$$

where $\mathcal{K}_{C}(\overline{\boldsymbol{F}})$ denotes the set of admissible deformation gradients $\boldsymbol{F}$ that satisfy the affine condition (1), as defined by (4), as well as the constraints

$$
\langle\boldsymbol{F}\rangle_{0}^{(s)}-\boldsymbol{F}^{(s)}=0, \quad \text { for } \quad s=1, \ldots, N
$$

This can be demonstrated by interchanging the stat over the variables $\boldsymbol{M}^{(s)}$ in expression (12) and the stat over $\boldsymbol{F}$ implicit in expression (9) for $\tilde{W}_{\boldsymbol{M}}$. In this context, it is important to note that the prescribed average deformations $\boldsymbol{F}^{(s)}$ are required to satisfy the constraint $\sum_{r=1}^{N} c_{0}^{(r)} \boldsymbol{F}^{(r)}=\overline{\boldsymbol{F}}$ for consistency with the overall macroscopic deformation $\overline{\boldsymbol{F}}$. (Note also that multiple solutions may become available in expression (12) at sufficiently large applied stretches, in which case care must be exercised in the interchange of the stat operations.) However, it is in general easier to first find an estimate for the variational problem (9), and then make use of the Legendre transformation (12) to obtain the corresponding estimate for $\tilde{W}_{\boldsymbol{F}}\left(\boldsymbol{F} ; \boldsymbol{F}^{(s)}\right)$. This is due to the fact that the deformation gradient in the variational problem (9) need not satisfy additional (unusual) constraints of the form (14). In this work, we will make use of this approach to handle the problem of prescribed rotations on particles.

For completeness, it is noted that $\tilde{W}_{\boldsymbol{M}}$ can also be expressed in terms of its Legendre dual $\tilde{W}_{\boldsymbol{F}}$ via

$$
\tilde{W}_{\boldsymbol{M}}\left(\overline{\boldsymbol{F}} ; \boldsymbol{M}^{(s)}\right)=\underset{\boldsymbol{F}^{(s)}}{\operatorname{stat}}\left[\sum_{r=1}^{N} c_{0}^{(r)} \boldsymbol{M}^{(r)} \cdot \boldsymbol{F}^{(r)}-\tilde{W}_{\boldsymbol{F}}\left(\overline{\boldsymbol{F}} ; \boldsymbol{F}^{(s)}\right)\right],
$$

whose stationary values are given by

$$
\boldsymbol{M}^{(s)}=\frac{1}{c_{0}^{(s)}} \frac{\partial}{\partial \boldsymbol{F}^{(s)}} \tilde{W}_{\boldsymbol{F}}\left(\overline{\boldsymbol{F}} ; \boldsymbol{F}^{(r)}\right), \quad \text { for } \quad s=1, \ldots, N
$$

This result of course corresponds to the inverse of expression (11) for the average deformation gradient $\overline{\boldsymbol{F}}^{(s)}=\boldsymbol{F}^{(s)}$ as a function of $\boldsymbol{M}^{(s)}$ (provided that the $\boldsymbol{F}^{(s)}$ satisfy the constraint $\sum_{r=1}^{N} c_{0}^{(r)} \boldsymbol{F}^{(r)}=\overline{\boldsymbol{F}}$ ). It should also be noted that the macroscopic constitutive relations in terms of $\tilde{W}_{\boldsymbol{M}}$ and $\tilde{W}_{\boldsymbol{F}}$ are respectively written

$$
\overline{\boldsymbol{S}}=\frac{\partial \tilde{W}_{\boldsymbol{M}}}{\partial \overline{\boldsymbol{F}}} \quad \text { and } \quad \overline{\boldsymbol{S}}=-\frac{\partial \tilde{W}_{\boldsymbol{F}}}{\partial \overline{\boldsymbol{F}}}
$$

\section{Second-order homogenization estimates for the effective energy and microstructure evolu- tion}

An estimate for the solution of the nonlinear variational problem (9) can be obtained by using the "secondorder" homogenization method of Ponte Castañeda (2002). The basic idea behind the "second-order" method 
is to make use of an appropriately chosen linear comparison composite (LCC) with the same microstructure as the nonlinear composite but with linear constitutive responses for the phases. One characteristic feature of this methods is that it is exact to the second order in heterogeneity contrast. In particular, in this paper we make use of the "generalized-secant" second-order (GSO) method (Lopez-Pamies and Ponte Castañeda, 2006a), which utilizes the second moments of the deformation gradient in the phases of the LCC to define the constitutive response of the phases in the LCC, and is known to provide fairly accurate results even for high-contrast composites.

Toward this end, we first remark that the variational problem (9) is expected to have a unique solution in the infinitesimal deformation regime (i.e., in the neighborhood of $\boldsymbol{F}=\boldsymbol{I}$ ). The continuation of this solution into the finite deformation regime is referred to here as the "principal" solution and may by obtained via the stationary variational problem

$$
\hat{W}_{\boldsymbol{M}}\left(\overline{\boldsymbol{F}} ; \boldsymbol{M}^{(r)}\right)=\operatorname{stat}_{\boldsymbol{F} \in \mathcal{K}(\overline{\boldsymbol{F}})}\left\langle W_{\boldsymbol{M}}(\mathbf{X}, \boldsymbol{F})\right\rangle_{0}=\operatorname{stat}_{\boldsymbol{F} \in \mathcal{K}(\overline{\boldsymbol{F}})} \sum_{r=1}^{N} c_{0}^{(r)}\left\langle W^{(r)}(\boldsymbol{F})+\boldsymbol{M}^{(r)} \cdot \boldsymbol{F}\right\rangle_{0}^{(r)} .
$$

The above "principal" solution continues to remain valid, such that $\tilde{W}_{\boldsymbol{M}}\left(\overline{\boldsymbol{F}} ; \boldsymbol{M}^{(r)}\right)=\hat{W}_{\boldsymbol{M}}\left(\overline{\boldsymbol{F}} ; \boldsymbol{M}^{(r)}\right)$, up until the onset of the first microscopic instability (Michel et al., 2010). After the material first encounters an instability, the principal solution is no longer relevant, and we have that $\tilde{W}_{\boldsymbol{M}}\left(\overline{\boldsymbol{F}} ; \boldsymbol{M}^{(r)}\right) \leq \hat{W}_{\boldsymbol{M}}\left(\overline{\boldsymbol{F}} ; \boldsymbol{M}^{(r)}\right)$. However, as recently argued by Lopez-Pamies and Ponte Castañeda (2006a), it is often the case that the first instability encountered is of the "macroscopic" type, and can be easily evaluated by checking the loss of strong ellipticity of the tangent moduli associated with the principal energy $\hat{W}_{\boldsymbol{M}}\left(\overline{\boldsymbol{F}} ; \boldsymbol{M}^{(r)}\right)$. Thus, in what follows the effective energy $\tilde{W}_{\boldsymbol{M}}\left(\overline{\boldsymbol{F}} ; \boldsymbol{M}^{(r)}\right)$ will be estimated by the principal solution $\hat{W}_{\boldsymbol{M}}\left(\overline{\boldsymbol{F}} ; \boldsymbol{M}^{(r)}\right)$, as obtained from the stationary variational problem (18). For a particularly relevant example of the computation of the homogenized stored-energy function $\tilde{W}$ beyond the onset of the first instability, the reader is referred to the recent work of Avazmohammadi and Ponte Castañeda (2015).

\subsection{GSO estimates for the principal solution under the application of eigenstresses}

As mentioned earlier the basic idea behind the second-order method is the construction of a fictitious LCC with the same microstructure as the nonlinear composite. Thus, we define the local stored-energy function of the LCC by means of

$$
W_{L C C}^{M}(\mathbf{X}, \boldsymbol{F})=\sum_{r=1}^{N} \Theta^{(r)}(\mathbf{X})\left[W_{L C C}^{(r)}(\boldsymbol{F})+\boldsymbol{M}^{(r)} \cdot \boldsymbol{F}\right],
$$

where $\Theta^{(r)}(\mathbf{X})$ are the characteristic functions describing the microstructure of the nonlinear composite and the quadratic functions $W_{L C C}^{(r)}(\boldsymbol{F})$ are given by Taylor approximations of the nonlinear phase energies $W^{(r)}$ about some "reference" deformations $\check{\boldsymbol{F}}^{(r)}$, i.e.,

$$
\begin{aligned}
W_{L C C}^{(r)}(\boldsymbol{F}): & =W^{(r)}\left(\check{\boldsymbol{F}}^{(r)}\right)+\mathcal{S}^{(r)}\left(\check{\boldsymbol{F}}^{(r)}\right) \cdot\left(\boldsymbol{F}-\check{\boldsymbol{F}}^{(r)}\right) \\
& +\frac{1}{2}\left(\boldsymbol{F}-\check{\boldsymbol{F}}^{(r)}\right) \cdot \mathbb{L}^{(r)}\left(\boldsymbol{F}-\check{\boldsymbol{F}}^{(r)}\right) \text {, where } \mathcal{S}^{(r)}(\boldsymbol{F}):=\frac{\partial}{\partial \boldsymbol{F}} W^{(r)}(\boldsymbol{F}) .
\end{aligned}
$$


Next, we define the corrector functions

$$
V^{(r)}\left(\check{\boldsymbol{F}}^{(r)}, \mathbb{L}^{(r)}\right)=\operatorname{stat}_{\hat{\boldsymbol{F}}^{(r)}}\left\{W^{(r)}\left(\hat{\boldsymbol{F}}^{(r)}\right)-W_{L C C}^{(r)}\left(\hat{\boldsymbol{F}}^{(r)}\right)\right\}
$$

which measure the nonlinearity of the stored energies $W^{(r)}$. Under appropriate conditions, the nonlinear energies $W^{(r)}$ may be written in terms of the linear energies $W_{L C C}^{(r)}$ and the corrector functions $V^{(r)}$ as follows,

$$
W^{(r)}(\boldsymbol{F})=\operatorname{stat}_{\mathbb{L}^{(r)}}\left\{W_{L C C}^{(r)}(\boldsymbol{F})+V^{(r)}\left(\check{\boldsymbol{F}}^{(r)}, \mathbb{L}^{(r)}\right)\right\} .
$$

Note that, this last expression is valid for arbitrary choices of the reference deformations $\check{\boldsymbol{F}}^{(r)}$.

Substituting $W^{(r)}$, as given by (22), into the variational problem (18) and interchanging the order of stationary operations with respect to $\boldsymbol{F}$ and $\mathbb{L}^{(r)}$, we arrive at

$$
\hat{W}_{\boldsymbol{M}}\left(\overline{\boldsymbol{F}}, \boldsymbol{M}^{(r)}\right)=\underset{\mathbb{L}^{(r)}}{\operatorname{stat}}\left\{\hat{W}_{L C C}^{\boldsymbol{M}}\left(\overline{\boldsymbol{F}} ; \boldsymbol{M}^{(r)}\right)+\sum_{s=1}^{N} c_{0}^{(s)} V^{(s)}\left(\check{\boldsymbol{F}}^{(s)}, \mathbb{L}^{(s)}\right)\right\} .
$$

In this last expression, $\hat{W}_{L C C}^{M}\left(\overline{\boldsymbol{F}} ; \boldsymbol{M}^{(r)}\right)$ is the effective energy of the LCC under the affine boundary conditions (1) and prescribed eigenstresses on the phases, i.e.,

$$
\hat{W}_{L C C}^{M}\left(\overline{\boldsymbol{F}} ; \boldsymbol{M}^{(r)}\right)=\operatorname{stat}_{\boldsymbol{F} \in \mathcal{K}(\overline{\boldsymbol{F}})} \sum_{r=1}^{N} c_{0}^{(r)}\left\langle W_{L C C}^{(r)}(\boldsymbol{F})+\boldsymbol{M}^{(r)} \cdot \boldsymbol{F}\right\rangle_{0}^{(r)} .
$$

Note that since $W_{L C C}^{(r)}$ on the right side of $(24)$ depends on $\check{\boldsymbol{F}}^{(r)}$ and $\mathbb{L}^{(r)}$, the effective energy $\hat{W}_{L C C}^{M}\left(\overline{\boldsymbol{F}} ; \boldsymbol{M}^{(r)}\right)$ will also depend on $\boldsymbol{F}^{(r)}$ and $\mathbb{L}^{(r)}$ for $r=1, \ldots, N$. It is important to remark that the stationary operation in $(23)$ is restricted to uniform modulus tensors $\mathbb{L}^{(r)}$. In general, it is possible to search for non-uniform modulus tensors $\mathbb{L}^{(r)}(\mathbf{X})$ that result in stationarity of the right side of (23); however, such possibilities are not explored here for simplicity.

The stationary conditions associated with (21) and (23) read, respectively, as

$$
\mathcal{S}^{(r)}\left(\hat{\boldsymbol{F}}^{(r)}\right)-\mathcal{S}^{(r)}\left(\check{\boldsymbol{F}}^{(r)}\right)=\mathbb{L}^{(r)}\left(\hat{\boldsymbol{F}}^{(r)}-\check{\boldsymbol{F}}^{(r)}\right)
$$

and

$$
\frac{\partial}{\partial \mathbb{L}^{(r)}} \hat{W}_{L C C}^{M}\left(\overline{\boldsymbol{F}} ; \boldsymbol{M}^{(r)}\right)+c_{0}^{(r)} \frac{\partial}{\partial \mathbb{L}^{(r)}} V^{(r)}\left(\check{\boldsymbol{F}}^{(r)}, \mathbb{L}^{(r)}\right)=0 .
$$

The condition (26) can be rewritten in terms of the fluctuations of the deformation gradient in phases of the LCC as follows

$$
\boldsymbol{C}_{L C C}^{(r)}=\left(\hat{\boldsymbol{F}}^{(r)}-\check{\boldsymbol{F}}^{(r)}\right) \otimes\left(\hat{\boldsymbol{F}}^{(r)}-\check{\boldsymbol{F}}^{(r)}\right)-\left(\overline{\boldsymbol{F}}_{L C C}^{(r)}-\check{\boldsymbol{F}}^{(r)}\right) \otimes\left(\overline{\boldsymbol{F}}_{L C C}^{(r)}-\check{\boldsymbol{F}}^{(r)}\right),
$$

where $\boldsymbol{C}_{L C C}^{(r)}:=\left\langle\left(\boldsymbol{F}-\overline{\boldsymbol{F}}_{L C C}^{(r)}\right) \otimes\left(\boldsymbol{F}-\overline{\boldsymbol{F}}_{L C C}^{(r)}\right)\right\rangle^{(r)}$ is the fluctuation of the deformation gradient in phase $r$ of the LCC.

Substituting $V^{(r)}$ from expression (21) and $\hat{W}_{L C C}^{M}$ from expression (24) into (23), and using the stationary condition (26), the GSO estimate for the effective energy of the composite of interest is obtained as follows

$$
\hat{W}_{\boldsymbol{M}}\left(\overline{\boldsymbol{F}} ; \boldsymbol{M}^{(r)}\right)=\sum_{s=1}^{N} c_{0}^{(s)}\left[W^{(s)}\left(\hat{F}^{(s)}\right)-\mathcal{S}^{(s)}\left(\check{\boldsymbol{F}}^{(s)}\right) \cdot\left(\hat{\boldsymbol{F}}^{(s)}-\overline{\boldsymbol{F}}_{L C C}^{(s)}\right)+\boldsymbol{M}^{(s)} \cdot \overline{\boldsymbol{F}}_{L C C}^{(s)}\right] .
$$


It is observed that the estimate (28) for the effective energy depends on the first and second moments of the deformation gradient in the phases of the LCC via $\overline{\boldsymbol{F}}_{L C C}^{(r)}$ and $\hat{\boldsymbol{F}}^{(r)}$, respectively. It is emphasized that (28) is valid independently of the choices for the reference deformation gradients $\check{\boldsymbol{F}}^{(r)}$, however certain physically based considerations must be satisfied for such choices (see Lopez-Pamies and Ponte Castañeda, 2006a, for more details).

In summary, having an expression for the effective energy of the LCC with prescribed eigenstresses for the phases, $\hat{W}_{L C C}^{M}\left(\overline{\boldsymbol{F}} ; \boldsymbol{M}^{(r)}\right)$, one can use (25) and (26) to solve for $\hat{\boldsymbol{F}}^{(r)}$. On the other hand, the averages of the deformation gradient in phases of the LCC may be computed as follows

$$
\overline{\boldsymbol{F}}_{L C C}^{(r)}=\frac{1}{c_{0}^{(r)}} \frac{\partial}{\partial \boldsymbol{M}^{(r)}} \hat{W}_{L C C}^{M}\left(\overline{\boldsymbol{F}} ; \boldsymbol{M}^{(s)}\right)
$$

Having obtained $\hat{\boldsymbol{F}}^{(r)}$ and $\overline{\boldsymbol{F}}_{L C C}^{(r)}$, one can use (28) to get an estimate for the effective energy of the nonlinear composite with prescribed eigenstresses in the phases.

\subsection{GSO estimates for the evolution of the microstructure}

The averages of the deformation gradient in phases of the nonlinear composite may be obtained by means of relations of the type (11). Thus, we have that

$$
\overline{\boldsymbol{F}}^{(s)}=\frac{1}{c_{0}^{(s)}} \frac{\partial}{\partial \boldsymbol{M}^{(s)}} \hat{W}_{\boldsymbol{M}}\left(\overline{\boldsymbol{F}} ; \boldsymbol{M}^{(r)}\right), \quad \text { for } \quad s=1, \ldots, N
$$

where $\hat{W}_{\boldsymbol{M}}\left(\overline{\boldsymbol{F}} ; \boldsymbol{M}^{(r)}\right)$ is the principal solution, as given by (23), for the nonlinear homogenization problem discussed in the previous subsection. In connection with (23), it is understood that $\hat{W}_{L C C}$ may in general depend on $\boldsymbol{M}^{(r)}$, but the corrector functions $V^{(r)}$ are independent of $\boldsymbol{M}^{(r)}$. Furthermore, based on the choices of the reference deformation gradients, they may also depend on the eigenstresses $\boldsymbol{M}^{(r)}$. For this reason, in the following calculations we assume that the reference deformation gradients $\check{\boldsymbol{F}}^{(r)}$ are, in general, functions of the eigenstresses $\boldsymbol{M}^{(r)}$.

Thus, taking derivative of $\hat{W}_{\boldsymbol{M}}\left(\overline{\boldsymbol{F}} ; \boldsymbol{M}^{(r)}\right)$, as given by (23), with respect to $\boldsymbol{M}^{(s)}$, it is found that

$$
\frac{\partial}{\partial \boldsymbol{M}^{(s)}} \hat{W}_{\boldsymbol{M}}\left(\overline{\boldsymbol{F}} ; \boldsymbol{M}^{(r)}\right)=\frac{\partial}{\partial \boldsymbol{M}^{(s)}} \hat{W}_{L C C}^{M}\left(\overline{\boldsymbol{F}} ; \boldsymbol{M}^{(r)}\right)+\sum_{r=1}^{N}\left[\frac{\partial \hat{W}_{L C C}}{\partial \check{\boldsymbol{F}}^{(r)}}+c_{0}^{(r)} \frac{\partial V^{(r)}}{\partial \check{\boldsymbol{F}}^{(r)}}\right] \cdot \frac{\partial \check{\boldsymbol{F}}^{(r)}}{\partial \boldsymbol{M}^{(s)}},
$$

where the notation

$$
\left[\frac{\partial}{\partial \check{\boldsymbol{F}}^{(r)}} \cdot \frac{\partial \check{\boldsymbol{F}}^{(r)}}{\partial \boldsymbol{M}^{(s)}}\right]_{p q}=\frac{\partial}{\partial \check{F}_{i j}^{(r)}} \frac{\partial \check{F}_{i j}^{(r)}}{\partial M_{p q}^{(s)}}
$$

is used. Note that in obtaining (31) we have used the fact that the right side of (23) is stationary with respect to $\mathbb{L}^{(s)}$. Next, using $(24)$ it can be shown that

$$
\frac{\partial \hat{W}_{L C C}^{M}}{\partial \check{\boldsymbol{F}}^{(r)}}=c_{0}^{(r)}\left(\mathbb{L}^{(r)}-\mathbb{L}_{t a n}^{(r)}\right)\left(\check{\boldsymbol{F}}^{(r)}-\overline{\boldsymbol{F}}_{L C C}^{(r)}\right)+\frac{c_{0}^{(r)}}{2} \mathbb{L}^{(r)} \cdot \frac{\partial \boldsymbol{C}_{L C C}^{(r)}}{\partial \check{\boldsymbol{F}}^{(r)}},
$$

where

$$
\mathbb{L}_{t a n}^{(r)}:=\frac{\partial^{2}}{\partial \boldsymbol{F}^{2}} W^{(r)}\left(\check{\boldsymbol{F}}^{(r)}\right)
$$


is a fourth-order tensor defining the tangent modulus tensor for phase $r$ of the composite evaluated at $\check{\boldsymbol{F}}^{(r)}$, and $\boldsymbol{C}_{L C C}^{(r)}$ is the fluctuation tensor of the deformation gradient in phase $r$ of the composite. Finally, the derivative of the corrector functions with respect to $\check{\boldsymbol{F}}^{(r)}$ may be obtained by using the definition (21), as follows

$$
\frac{\partial V^{(r)}}{\partial \check{\boldsymbol{F}}^{(r)}}=\left(\mathbb{L}^{(r)}-\mathbb{L}_{t a n}^{(r)}\right) \cdot\left(\hat{\boldsymbol{F}}^{(r)}-\check{\boldsymbol{F}}^{(r)}\right),
$$

where use has been made of the fact that the right side of (21) is stationary with respect to $\hat{\boldsymbol{F}}^{(r)}$.

Substituting expressions (33) and (35) into (31), we obtain

$$
\frac{\partial}{\partial \boldsymbol{M}^{(s)}} \hat{W}\left(\overline{\boldsymbol{F}} ; \boldsymbol{M}^{(r)}\right)=\frac{\partial}{\partial \boldsymbol{M}^{(s)}} \hat{W}_{L C C}^{M}\left(\overline{\boldsymbol{F}} ; \boldsymbol{M}^{(r)}\right)+\sum_{r=1}^{N} c_{0}^{(r)} \boldsymbol{\rho}^{(r)} \cdot \frac{\partial \check{\boldsymbol{F}}^{(r)}}{\partial \boldsymbol{M}^{(s)}},
$$

where

$$
\boldsymbol{\rho}^{(r)}:=\left(\mathbb{L}^{(r)}-\mathbb{L}_{t a n}^{(r)}\right)\left(\hat{\boldsymbol{F}}^{(r)}-\overline{\boldsymbol{F}}_{L C C}^{(r)}\right)+\frac{1}{2} \mathbb{L}^{(r)} \cdot \frac{\partial \boldsymbol{C}_{L C C}^{(r)}}{\partial \check{\boldsymbol{F}}^{(r)}} .
$$

Thus, the average of the deformation gradient in phase $s$ of the nonlinear composite can be obtained in terms of $\overline{\boldsymbol{F}}_{L C C}^{(s)}$ as follows

$$
\overline{\boldsymbol{F}}^{(s)}=\overline{\boldsymbol{F}}_{L C C}^{(s)}+\sum_{r=1}^{N} \frac{c_{0}^{(r)}}{c_{0}^{(s)}} \boldsymbol{\rho}^{(r)} \cdot \frac{\partial \check{\boldsymbol{F}}^{(r)}}{\partial \boldsymbol{M}^{(s)}}
$$

As it is evident from this last expression, in general, the averages of the deformation gradient in phases of the nonlinear composite are different from the corresponding averages in the LCC.

\section{GSO estimates for two-phase composites in the presence of an eigenstress}

In this section we specialize the general estimates of section 4 for the case of two-phase particulate composites. In particular, we consider composites consisting of a soft elastomeric matrix phase with storedenergy function $W^{(1)}(\boldsymbol{F})$, occupying the volume $\Omega_{0}^{(1)}$ in the reference configuration, and an inclusion phase with stored-energy function $W^{(2)}(\boldsymbol{F})$, occupying the volume $\Omega_{0}^{(2)}$ in the reference configuration. The inclusions have identical ellipsoidal shapes and are distributed with ellipsoidal symmetry (Willis, 1977; Ponte Castañeda and Willis, 1995). The initial volume fraction of the inclusions is denoted by $c_{0}$, such that

$$
c_{0}^{(1)}=1-c_{0}, \quad \text { and } \quad c_{0}^{(2)}=c_{0} .
$$

In addition, an eigenstress $\boldsymbol{M}^{(2)}$ is applied to the inclusion phase, such that

$$
\boldsymbol{M}(\mathbf{X})=\Theta^{(2)}(\mathbf{X}) M^{(2)}
$$

where, as usual, $\Theta^{(2)}$ is the characteristic function of the inclusion phase.

Recall that the computation of the effective energy of the nonlinear composites, as given by (28), requires estimates for the first and second moments of the deformation gradient field in the phases of the corresponding LCC. Such estimates for two-phase LCCs with linear phase energies of the form (20) may be obtained by using appropriate generalizations of the Levin (1967) relations for thermoelastic composites in combination 
with the Hashin-Shtrikman-type estimates of Ponte Castañeda and Willis (1995) for the above-described particulate microstructures (see Appendix B for the details). Thus, making the substitutions

$$
\begin{aligned}
\boldsymbol{T}^{(1)} & =\mathcal{S}^{(1)}-\mathbb{L}^{(1)} \check{\boldsymbol{F}}^{(1)}, \quad \boldsymbol{T}^{(2)}=\mathcal{S}^{(2)}-\mathbb{L}^{(2)} \check{\boldsymbol{F}}^{(2)}+\boldsymbol{M}^{(2)}, \quad \text { and } \\
f^{(r)} & =W^{(r)}\left(\check{\boldsymbol{F}}^{(r)}\right)-\mathcal{S}^{(r)} \cdot \check{\boldsymbol{F}}^{(r)}+\frac{1}{2} \check{\boldsymbol{F}}^{(r)} \cdot \mathbb{L}^{(r)} \check{\boldsymbol{F}}^{(r)}, \quad \text { for } \quad r=1,2,
\end{aligned}
$$

generated from expressions (19) and (20), into expression (B.17) of Appendix B for $\overline{\boldsymbol{F}}_{L C C}^{(2)}$, we arrive at

$$
\overline{\boldsymbol{F}}-\overline{\boldsymbol{F}}_{L C C}^{(2)}=\left(1-c_{0}\right) \mathbb{P}\left[\mathbb{L}^{(1)}\left(\check{\boldsymbol{F}}^{(1)}-\overline{\boldsymbol{F}}_{L C C}^{(2)}\right)+\boldsymbol{M}^{(2)}-\mathcal{S}^{(1)}+\mathcal{S}^{(2)}\right],
$$

while $\boldsymbol{C}_{L C C}^{(2)}=0$, because of the Hashin-Shtrikman type estimates used in the computation of the effective energy. Note that in obtaining (42) we made the replacement $\check{\boldsymbol{F}}^{(2)}=\overline{\boldsymbol{F}}_{L C C}^{(2)}$. Having obtained $\overline{\boldsymbol{F}}_{L C C}^{(2)}$, we can easily obtain the corresponding average in the matrix phase via

$$
\overline{\boldsymbol{F}}_{L C C}^{(1)}=\frac{1}{1-c_{0}}\left(\overline{\boldsymbol{F}}-c_{0} \overline{\boldsymbol{F}}_{L C C}^{(2)}\right) .
$$

To obtain the unknowns $\hat{\boldsymbol{F}}^{(1)}$ and $\hat{\boldsymbol{F}}^{(2)}$, we make use of the stationary conditions (25) and (26) (or (27)). First, we recall that $C_{L C C}^{(2)}=0$, and therefore the stationary condition (27) for phase 2 implies that

$$
\hat{\boldsymbol{F}}^{(2)}=\overline{\boldsymbol{F}}_{L C C}^{(2)} \text {. }
$$

Using (44), the stationary condition (25) for $r=2$, can be shown to reduce to an equation to the effect that the modulus $\mathbb{L}^{(2)}$ of phase 2 in the LCC is given by the tangent modulus of nonlinear phase 2 , evaluated at $\check{\boldsymbol{F}}^{(2)}$. On the other hand, for $r=1$, the stationary condition (25) reduces to

$$
\mathcal{S}^{(1)}\left(\hat{\boldsymbol{F}}^{(1)}\right)-\mathcal{S}^{(1)}\left(\check{\boldsymbol{F}}^{(1)}\right)=\mathbb{L}^{(1)}\left(\hat{\boldsymbol{F}}^{(1)}-\check{\boldsymbol{F}}^{(1)}\right),
$$

while the stationary condition (26) for phase 1 of the composite can be rewritten as

$$
\left(\hat{\boldsymbol{F}}^{(1)}-\check{\boldsymbol{F}}^{(1)}\right) \otimes\left(\hat{\boldsymbol{F}}^{(1)}-\check{\boldsymbol{F}}^{(1)}\right)=\frac{2}{1-c_{0}} \frac{\partial \hat{W}_{L C C}^{M}}{\partial \mathbb{L}^{(1)}}
$$

An expression for $\hat{W}_{L C C}^{M}$ may be obtained by making the substitutions (41) into (B.2). Taking the derivative of the resulting expression with respect to $\mathbb{L}^{(1)}$, the right side of (46) can be further simplified as follows

$$
\begin{aligned}
\frac{2}{1-c_{0}} \frac{\partial \hat{W}_{L C C}^{M}}{\partial \mathbb{L}^{(1)}} & =\frac{1}{1-c_{0}}\left(\check{\boldsymbol{F}}^{(1)}-\overline{\boldsymbol{F}}\right) \otimes\left(\check{\boldsymbol{F}}^{(1)}-\overline{\boldsymbol{F}}\right) \\
& -\frac{c_{0}}{1-c_{0}}\left(\check{\boldsymbol{F}}^{(1)}-\overline{\boldsymbol{F}}_{L C C}^{(2)}\right) \otimes\left(\check{\boldsymbol{F}}^{(1)}-\overline{\boldsymbol{F}}_{L C C}^{(2)}\right) \\
& -\frac{c_{0}}{\left(1-c_{0}\right)^{2}}\left(\overline{\boldsymbol{F}}-\overline{\boldsymbol{F}}_{L C C}^{(2)}\right) \cdot\left[\mathbb{P}^{-1} \frac{\partial \mathbb{P}}{\partial \mathbb{L}^{(1)}} \mathbb{P}^{-1}\right]\left(\overline{\boldsymbol{F}}-\overline{\boldsymbol{F}}_{L C C}^{(2)}\right) .
\end{aligned}
$$

It is remarked here that the right side of (47) is exactly identical to the corresponding expression in LopezPamies and Ponte Castañeda (2006b), with the only difference that now the average deformation gradient in phase two of the LCC depends on the eigenstress $\boldsymbol{M}^{(2)}$, as is evident from (42). 
In summary, for a given eigenstress $\boldsymbol{M}^{(2)}$, equations (42)-(46) can be solved to obtain $\overline{\boldsymbol{F}}_{L C C}^{(r)}$ and $\hat{\boldsymbol{F}}^{(r)}$ $(r=1,2)$, and $\mathbb{L}^{(1)}$. The resulting quantities, along with the appropriate choice for the reference deformation gradient $\check{\boldsymbol{F}}^{(1)}$, can then be inserted into the general expression (28) to obtain an estimate for the effective stored-energy of the two-phase nonlinear composite, i.e.,

$\hat{W}_{\boldsymbol{M}}\left(\overline{\boldsymbol{F}} ; \boldsymbol{M}^{(2)}\right)=\left(1-c_{0}\right)\left[W^{(1)}\left(\hat{\boldsymbol{F}}^{(1)}\right)-\mathcal{S}^{(1)}\left(\check{\boldsymbol{F}}^{(1)}\right) \cdot\left(\hat{\boldsymbol{F}}^{(1)}-\overline{\boldsymbol{F}}_{L C C}^{(1)}\right)\right]+c_{0}\left[W^{(2)}\left(\overline{\boldsymbol{F}}_{L C C}^{(2)}\right)+\boldsymbol{M}^{(2)} \cdot \overline{\boldsymbol{F}}_{L C C}^{(2)}\right]$.

The average deformation gradient in the phases of the nonlinear composite may then be obtained by means of the general expression (38). Thus, using the fact that $\boldsymbol{C}_{L C C}^{(2)}=0$ and making the choice $\check{\boldsymbol{F}}^{(1)}=\overline{\boldsymbol{F}}$ for the reference deformation gradient in phase 1 , it can be shown that

$$
\overline{\boldsymbol{F}}^{(1)}=\overline{\boldsymbol{F}}_{L C C}^{(1)} \quad \text { and } \quad \overline{\boldsymbol{F}}^{(2)}=\overline{\boldsymbol{F}}_{L C C}^{(2)} .
$$

In other words, for the special case of two-phase composites and when the fluctuation of the deformation gradient is identically zero in the inclusion phase, the averages of the deformation gradient in the phases of the nonlinear composite are exactly equal to the corresponding averages in the LCC. Note that this result is a special property of the Hashin-Shtrikman type estimates used in the computation of $\tilde{\mathbb{L}}$ in the LCC, and may not be true for other type of estimates (as can be seen from general expression (38) in section 4).

Specialization to two-phase particulate composites consisting of rigid inclusions

The above general result for two-phase particulate composites with compliant phases can be easily specialized for the case of composites with rigid inclusions. Thus, for two-phase composites reinforced by a rigid inclusion phase, we have that

$$
\overline{\boldsymbol{F}}^{(2)}=\overline{\boldsymbol{F}}_{L C C}^{(2)}=\overline{\boldsymbol{R}}^{(2)} \quad \text { and } \quad \overline{\boldsymbol{F}}^{(1)}=\overline{\boldsymbol{F}}_{L C C}^{(1)}=\frac{1}{1-c_{0}}\left(\overline{\boldsymbol{F}}-c_{0} \overline{\boldsymbol{R}}^{(2)}\right),
$$

where we used expressions (49) and the fact that rigid inclusions can only undergo rigid body rotations, as characterized by the macroscopic rotation $\overline{\boldsymbol{R}}^{(2)}$. Furthermore, we have that $\hat{\boldsymbol{F}}^{(2)}=\overline{\boldsymbol{F}}_{L C C}^{(2)}=\overline{\boldsymbol{R}}^{(2)}$. Then, making use of the above expressions for $\hat{\boldsymbol{F}}^{(2)}, \overline{\boldsymbol{F}}_{L C C}^{(1)}$, and $\overline{\boldsymbol{F}}_{L C C}^{(2)}$ in (48), we arrive at the following expression for the effective stored-energy function of the two-phase, rigidly reinforced composites

$$
\hat{W}_{\boldsymbol{M}}\left(\overline{\boldsymbol{F}} ; \boldsymbol{M}^{(2)}\right)=\left(1-c_{0}\right) W^{(1)}\left(\hat{\boldsymbol{F}}^{(1)}\right)+\mathcal{S}^{(1)} \cdot\left[\overline{\boldsymbol{F}}-c_{0} \overline{\boldsymbol{R}}^{(2)}-\left(1-c_{0}\right) \hat{\boldsymbol{F}}^{(1)}\right]+c_{0} \boldsymbol{M}^{(2)} \cdot \overline{\boldsymbol{R}}^{(2)},
$$

Note that in this expression we are using the shorthanded notation $\mathcal{S}^{(1)}=\mathcal{S}^{(1)}(\overline{\boldsymbol{F}})$. On the other hand, in the limit as the inclusions become rigid, equation (42) reduces to

$$
\begin{aligned}
& \left(1-c_{0}\right)\left[\left(\overline{\boldsymbol{R}}^{(2)}\right)^{T} \boldsymbol{M}^{(2)}-\left(\boldsymbol{M}^{(2)}\right)^{T} \overline{\boldsymbol{R}}^{(2)}\right]=\left(1-c_{0}\right)\left[\left(\overline{\boldsymbol{R}}^{(2)}\right)^{T} \mathcal{S}^{(1)}-\left(\mathcal{S}^{(1)}\right)^{T} \overline{\boldsymbol{R}}^{(2)}\right] \\
& +\left(\overline{\boldsymbol{R}}^{(2)}\right)^{T}\left[\mathbb{D}\left(\overline{\boldsymbol{F}}-\overline{\boldsymbol{R}}^{(2)}\right)\right]-\left[\mathbb{D}\left(\overline{\boldsymbol{F}}-\overline{\boldsymbol{R}}^{(2)}\right)\right]^{T}\left(\overline{\boldsymbol{R}}^{(2)}\right),
\end{aligned}
$$

where $\mathbb{D}:=\mathbb{P}^{-1}-\left(1-c_{0}\right) \mathbb{L}^{(1)}$. In obtaining the above identity we have used the fact that

$$
\left[\left(\overline{\boldsymbol{R}}^{(2)}\right)^{T} \mathcal{S}^{(2)}\left(\overline{\boldsymbol{R}}^{(2)}\right)-\left(\mathcal{S}^{(2)}\left(\overline{\boldsymbol{R}}^{(2)}\right)\right)^{T} \overline{\boldsymbol{R}}^{(2)}\right]=0,
$$


in the limit as the particles become rigid (see Lopez-Pamies and Ponte Castañeda, 2006a, for more details).

Finally, the fluctuation equation (46) for the case of rigid inclusions can be rewritten as follows

$$
\left(\hat{\boldsymbol{F}}^{(1)}-\check{\boldsymbol{F}}^{(1)}\right) \otimes\left(\hat{\boldsymbol{F}}^{(1)}-\check{\boldsymbol{F}}^{(1)}\right)=\frac{c_{0}}{\left(1-c_{0}\right)^{2}}\left(\overline{\boldsymbol{F}}-\overline{\boldsymbol{R}}^{(2)}\right) \cdot \frac{\partial \mathbb{D}}{\partial \mathbb{L}^{(1)}}\left(\overline{\boldsymbol{F}}-\overline{\boldsymbol{R}}^{(2)}\right),
$$

where the fourth-order tensor $\mathbb{D}$ is as defined earlier. (Note that, as will be seen later, only certain traces of this expression can be satisfied.)

\section{Incompressible elastomers reinforced by rigid fibers undergoing plane-strain deformations}

In this section we apply the general results of the previous section to elastomers reinforced with one family of long rigid fibers with general elliptical shapes for the cross-section under 2D plane-strain deformations. Thus, as shown in Fig. 1, we consider a two-phase composite consisting of a matrix phase, denoted by the

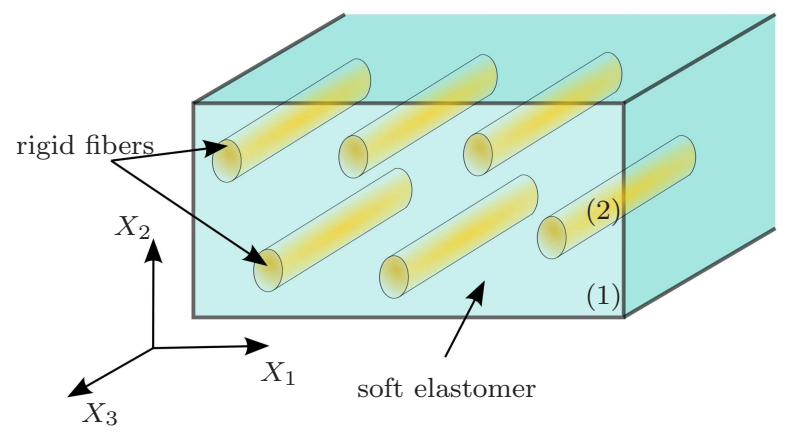

(a)

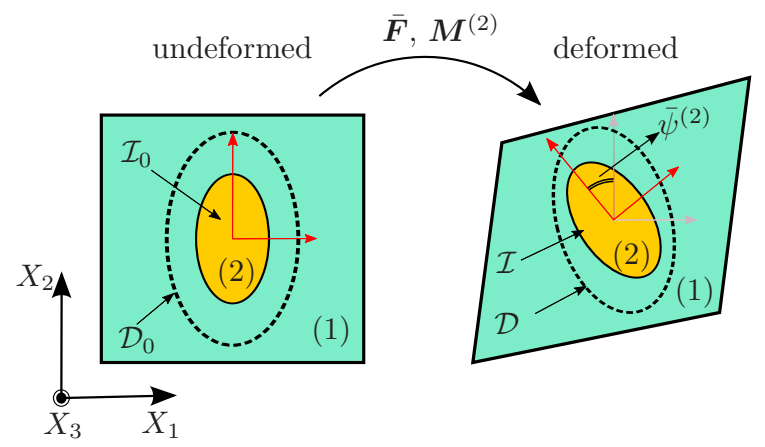

(b)

Figure 1: Schematic representation of two-phase fiber-reinforced composites.(a) The composite sample consisting of very long rigid fibers, embedded firmly in an elastomeric phase. (b) The microstructural details of the composite in the reference and deformed configurations.

superscript "1", and a fiber phase, denoted by the superscript "2". The fibers (in the reference configuration) are aligned with the $X_{3}$ direction of the laboratory axis $\left\{X_{1}, X_{2}, X_{3}\right\}$ and are assumed to be very long such that their geometry is completely described by the shape of their cross-sections. In this work we consider elliptical shapes for the cross-section of the fibers as characterized by the ellipses

$$
\mathcal{I}_{0}=\left\{\mathbf{X}=\left(X_{1}, X_{2}\right), \text { such that }\left|\boldsymbol{Z}_{0}^{T} \mathbf{X}\right| \leq 1\right\},
$$

where $\boldsymbol{Z}_{0}$ is a second-order tensor describing the shape (in the reference configuration) of the fibers. Furthermore, we assume that the in-plane axes of the fibers are aligned with the $X_{1}$ and $X_{2}$ directions, such that

$$
\boldsymbol{Z}_{0}=\operatorname{diag}(a, w a)
$$

with $w$ being the aspect ratio of the fibers. As mentioned earlier, the fibers are distributed with "elliptical symmetry" (Ponte Castañeda and Willis, 1995) in the $X_{1}-X_{2}$ plane. This means that the two-point probability function for finding two fibers separated by a vector $\mathbf{Y}=\left(Y_{1}, Y_{2}\right)$ depends on $\mathbf{Y}$ only through the 
combination $\left|\left(\boldsymbol{D}_{0}^{-T} \mathbf{Y}\right)\right|$, where $\boldsymbol{D}_{0}$ is a symmetric, second-order tensor serving to define a "distributional ellipse" in the reference configuration via

$$
\mathcal{D}_{0}=\left\{\mathbf{X}=\left(X_{1}, X_{2}\right), \text { such that }\left|\boldsymbol{D}_{0}^{-T} \mathbf{X}\right| \leq 1\right\} .
$$

Note that statistical isotropy can be achieved by setting $\boldsymbol{D}_{0}=\boldsymbol{I}$, such that the two-point probability function depends on $\mathbf{Y}$ only via $|\mathbf{Y}|$, and the distributional ellipse becomes a circle. In this paper, for simplicity, we assume that the shape of the distributional ellipse in the reference configuration is identical to the shape of the fibers such that $\boldsymbol{D}_{0}=Z_{0}$.

For the constitutive response of the matrix phase, we consider an incompressible, isotropic, stored energy, which is obtained by taking the limit as $\kappa \rightarrow \infty$ of the general (compressible) energy

$$
W^{(1)}(\boldsymbol{F})=g(I)+h(J)+\frac{\kappa-\mu}{2}(J-1)^{2}, \quad \text { where } \quad I:=\boldsymbol{F} \cdot \boldsymbol{F}, \quad J:=\operatorname{det} \boldsymbol{F} .
$$

A simple special case of the above general functional form for $W^{(1)}$ is

$$
W^{(1)}(\boldsymbol{F})=-\frac{\mu J_{m}}{2} \ln \left(1-\frac{I-2}{J_{m}}\right)-\mu \ln J+\left(\frac{\kappa-\mu}{2}-\frac{\mu}{J_{m}}\right)(J-1)^{2},
$$

which corresponds to the Gent (1996) model. Note that this model accounts for the limiting chain extensibility of the elastomer, and in the limit as $J_{m} \rightarrow \infty$ reduces to a Neo-Hookean model. The rigid fibers are described by a stored-energy function of the form

$$
W^{(2)}(\boldsymbol{F})=0, \quad \text { when } \quad \boldsymbol{F}=\overline{\boldsymbol{R}}^{(2)}, \quad \text { and } \quad W^{(2)}(\boldsymbol{F})=\infty, \quad \text { otherwise. }
$$

Finally, an eigenstress $\boldsymbol{M}^{(2)}$ is applied on the fibers, with the following representation in the laboratory coordinate system

$$
\left[\boldsymbol{M}^{(2)}\right]=\left[\begin{array}{cc}
M_{11}^{(2)} & M_{12}^{(2)} \\
M_{21}^{(2)} & M_{22}^{(2)}
\end{array}\right]
$$

Before providing the results for the incompressibility limit (i.e., $\kappa \rightarrow \infty$ ), it is useful to spell out the details of calculations when the composite sample is constrained to undergo 2D deformations in the plane perpendicular to the long axis of the fibers. Thus, given the laboratory axes $\left\{X_{1}, X_{2}, X_{3}\right\}$, we assume that the macroscopic deformation of the composite is constrained to be in the $X_{1}-X_{2}$ plane, such that

$$
[\overline{\boldsymbol{F}}]=[\overline{\boldsymbol{R}}][\overline{\boldsymbol{U}}] \quad \text { and } \quad[\overline{\boldsymbol{U}}]=[\overline{\boldsymbol{Q}}][\overline{\boldsymbol{\Lambda}}][\overline{\boldsymbol{Q}}]^{T},
$$

where

$$
[\overline{\boldsymbol{R}}]=\left[\begin{array}{cc}
\cos \bar{\psi} & -\sin \bar{\psi} \\
\sin \bar{\psi} & \cos \bar{\psi}
\end{array}\right], \quad[\overline{\boldsymbol{Q}}]=\left[\begin{array}{cc}
\cos \bar{\theta} & -\sin \bar{\theta} \\
\sin \bar{\theta} & \cos \bar{\theta}
\end{array}\right], \quad \text { and } \quad[\overline{\mathbf{\Lambda}}]=\left[\begin{array}{cc}
\bar{\lambda}_{1} & 0 \\
0 & \bar{\lambda}_{2}
\end{array}\right] .
$$

Here the angle $\bar{\psi}$ denotes the macroscopic in-plane rotation of the sample, $\bar{\theta}$ denotes the in-plane loading angle, and $\bar{\lambda}_{1}$ and $\bar{\lambda}_{2}$ denote the (in-plane) principal stretches. The average rotation of the fibers $\overline{\boldsymbol{R}}^{(2)}$ for the case of $2 \mathrm{D}$ deformations can be written as

$$
\left[\overline{\boldsymbol{R}}^{(2)}\right]=\left[\begin{array}{cc}
\cos \bar{\psi}^{(2)} & -\sin \bar{\psi}^{(2)} \\
\sin \bar{\psi}^{(2)} & \cos \bar{\psi}^{(2)}
\end{array}\right]
$$


where $\bar{\psi}^{(2)}$ denotes the average in-plane rotation of the fibers. Next, we make use the prescription (LopezPamies and Ponte Castañeda, 2006a)

$$
L_{i j k l}^{(1)}=Q_{r m}^{(1)} Q_{j n}^{(1)} Q_{s p}^{(1)} Q_{l q}^{(1)} R_{i r}^{(1)} R_{k s}^{(1)} L_{m n p q}^{*}
$$

for the modulus tensor $\mathbb{L}^{(1)}$, which is consistent with the objectivity and material symmetry restrictions of the tangent modulus tensor, to further simplify the stationary condition (47). For the special case of 2D deformations, $\mathbb{L}^{*}$ is taken (see Lopez-Pamies and Ponte Castañeda, 2006b, for more details) to be orthotropic, i.e,

where

$$
\left[\mathbb{L}^{*}\right]=\left[\begin{array}{cccc}
L_{1111}^{*} & L_{1122}^{*} & 0 & 0 \\
L_{1122}^{*} & L_{2222}^{*} & 0 & 0 \\
0 & 0 & L_{1212}^{*} & L_{1221}^{*} \\
0 & 0 & L_{1221}^{*} & L_{2121}^{*}
\end{array}\right]
$$

$$
L_{1111}^{*}:=l_{1}, L_{2222}^{*}:=l_{2}, L_{1212}^{*}=L_{2121}^{*}:=l_{3}, L_{1122}^{*}:=l_{4}, \text { and } L_{1221}^{*}:=\sqrt{\left(l_{1}-l_{3}\right)\left(l_{2}-l_{3}\right)}-l_{4} .
$$

Note that, as pointed out by Lopez-Pamies and Ponte Castañeda (2006a), the choice (66) and (67) for $\mathbb{L}^{*}$ is a bit special; other more general prescriptions are possible. However, this choice has the advantage that it allows for closed-form analytical solutions, and for this reason it will be used here. With the above choices for the components of $\mathbb{L}^{*}$ and the following definition for the second-order tensor $\boldsymbol{Y}$

$$
\boldsymbol{Y}:=\overline{\boldsymbol{Q}}^{T} \overline{\boldsymbol{R}}^{T}\left(\hat{\boldsymbol{F}}^{(1)}-\overline{\boldsymbol{F}}\right) \overline{\boldsymbol{Q}}
$$

the fluctuation equations (46) and (47) for the special case of plane-strain deformations can be re-written as

$$
\begin{aligned}
Y_{11}^{2}+2 f_{1} Y_{12} Y_{21} & =\frac{2}{1-c_{0}} \frac{\partial \hat{W}_{L C C}}{\partial l_{1}}:=k_{1} \\
Y_{22}^{2}+2 f_{2} Y_{12} Y_{21} & =\frac{2}{1-c_{0}} \frac{\partial \hat{W}_{L C C}}{\partial l_{2}}:=k_{2} \\
Y_{11}^{2}+Y_{22}^{2}+2 f_{3} Y_{12} Y_{21} & =\frac{2}{1-c_{0}} \frac{\partial \hat{W}_{L C C}}{\partial l_{3}}:=k_{3} \\
2 Y_{11} Y_{22}-2 Y_{12} Y_{21} & =\frac{2}{1-c_{0}} \frac{\partial \hat{W}_{L C C}}{\partial l_{4}}:=k_{4},
\end{aligned}
$$

where the following definitions have been used

$$
f_{1}:=\frac{\partial L_{1221}^{*}}{\partial l_{1}}, \quad f_{2}:=\frac{\partial L_{1221}^{*}}{\partial l_{2}}, \quad \text { and } \quad f_{3}:=\frac{\partial L_{1221}^{*}}{\partial l_{3}} .
$$

Equations (69) can be solved for $Y_{11}$ and $Y_{22}$ to obtain

$$
Y_{11}= \pm \frac{f_{1} k_{4}+k_{1}}{\sqrt{4 f_{1}^{2} k_{2}+2 f_{1} k_{4}+k_{1}}} \quad \text { and } \quad Y_{22}= \pm \frac{2 f_{1} k_{2}+k_{4} / 2}{\sqrt{4 f_{1}^{2} k_{2}+2 f_{1} k_{4}+k_{1}}}
$$

Note that the combinations $Y_{12} Y_{21}$ and $Y_{12}^{2}+Y_{21}^{2}$ can then be uniquely determined in terms of $Y_{11}$ and $Y_{22}$. Next, each of the two solutions in (71) can be used to obtain $\hat{\boldsymbol{F}}^{(1)}=\overline{\boldsymbol{F}}+\overline{\boldsymbol{R}} \overline{\boldsymbol{Q}} \boldsymbol{Y} \overline{\boldsymbol{Q}}^{T}$, which in turn 
can be substituted into the stationary condition (45) to obtain a set of four nonlinear equations. These four equations, along with equation (52) for the average rotation of the fibers $\overline{\boldsymbol{R}}^{(2)}$, form a closed system of five nonlinear equations for the five unknowns of the problem, namely $l_{1}, l_{2}, l_{3}, l_{4}$, and $\bar{\psi}^{(2)}$. The above system of five nonlinear equations can be solved numerically to determine $\overline{\boldsymbol{R}}^{(2)}$ and $\hat{\boldsymbol{F}}^{(1)}$, for a given macroscopic deformation $\overline{\boldsymbol{F}}$ and eigenstress $\boldsymbol{M}^{(2)}$. In turn, their substitution into (51) provides an estimate for $\hat{W}_{\boldsymbol{M}}\left(\overline{\boldsymbol{F}} ; \boldsymbol{M}^{(2)}\right)$.

\subsection{Computation of $\hat{W}_{\boldsymbol{M}}\left(\overline{\boldsymbol{F}} ; \boldsymbol{M}^{(2)}\right)$ in the incompressibility limit}

Expression (51) for the effective energy $\hat{W}_{\boldsymbol{M}}\left(\overline{\boldsymbol{F}} ; \boldsymbol{M}^{(2)}\right)$ can be significantly simplified in the limit as $\kappa \rightarrow \infty$ (i.e., the incompressibility limit) by making use of the asymptotic analysis of Lopez-Pamies and Ponte Castañeda (2006b) in terms of the small parameter $\delta:=\kappa^{-1 / 3}$. In particular, this requires finding the leading (zeroth) order term in the expansions of $\boldsymbol{Y}$ and $\overline{\boldsymbol{R}}^{(2)}$ by using the secant condition (45), the fluctuation conditions (69), and the evolution equation (52), in the context of $2 \mathrm{D}$ deformations of the form (62). Toward this end, we note that the secant condition (45), and the fluctuation conditions (69), which are special cases of conditions (46) and (47), have no explicit dependence on the eigenstress $\boldsymbol{M}^{(2)}$. Thus, the leading-order terms in the expansion of $\boldsymbol{Y}$ can be shown to be exactly identical to the corresponding terms in Lopez-Pamies and Ponte Castañeda (2006b), where there is no eigenstress, i.e.,

$$
\begin{aligned}
Y_{11} & =\frac{c_{0}}{1-c_{0}}\left(\bar{\lambda}-\cos \varphi_{0}\right)+\mathcal{O}(\delta) \\
Y_{22} & =\frac{c_{0}}{1-c_{0}}\left(\bar{\lambda}^{-1}-\cos \varphi_{0}\right)+\mathcal{O}(\delta) \\
Y_{12} Y_{21} & =\frac{c_{0}}{\bar{\lambda}\left(1-c_{0}\right)^{2}}\left[\left(2-c_{0}\right) \bar{\lambda}-\left(\bar{\lambda}^{2}+1\right) \cos \varphi_{0}+c_{0} \bar{\lambda} \cos ^{2} \varphi_{0}\right]+\mathcal{O}(\delta) \\
Y_{12}^{2}+Y_{21}^{2} & =\frac{c_{0}}{2 w \bar{\lambda}\left(1-c_{0}\right)^{2}}\left\{-4[1+(w-1) w]\left(\bar{\lambda}+\bar{\lambda}^{3}\right) \cos \varphi_{0}\right. \\
& +2\left[w\left(c_{0}-4\right) \bar{\lambda}^{2}+\left(1+w^{2}\right)\left(1+\bar{\lambda}^{2}\right)^{2}-w c \bar{\lambda}^{2} \cos \left(2 \varphi_{0}\right)\right] \\
& \left.-2\left(w^{2}-1\right)\left(\bar{\lambda}^{4}-1\right) \cos (2 \bar{\theta}) \sin ^{2} \varphi_{0}+\left(w^{2}-1\right)\left(\bar{\lambda}^{4}-1\right) \sin (2 \bar{\theta}) \sin \left(2 \varphi_{0}\right)\right\}+\mathcal{O}(\delta) .
\end{aligned}
$$

In the above expressions, $\varphi_{0}$ is the leading order term in the expansion of the relative in-plane rotation $\varphi:=\bar{\psi}^{(2)}-\bar{\psi}$ of the fibers, such that $\varphi=\varphi_{0}+\mathcal{O}(\delta)$. Using the zeroth order terms for the components of the tensor $\boldsymbol{Y}$, as given in (72), along with the definition (68), we can obtain the leading-order terms for the components of the matrix $\hat{\boldsymbol{F}}^{(1)}$. In particular, the expansion of $\hat{I}^{(1)}=\hat{\boldsymbol{F}}^{(1)} \cdot \hat{\boldsymbol{F}}^{(1)}$ to zeroth order in the small parameter $\delta$ yields

$$
\begin{aligned}
\hat{I}_{0}^{(1)} & =\frac{c_{0}\left(1+\bar{\lambda}^{2}\right)^{2}+\left[1+2\left(c_{0}-2\right) c_{0} \bar{\lambda}^{2}+\bar{\lambda}^{4}\right] w+c_{0}\left(1+\bar{\lambda}^{2}\right)^{2} w^{2}}{\left(1-c_{0}\right)^{2} \bar{\lambda}^{2} w} \\
& -\frac{c_{0}\left(\bar{\lambda}^{4}-1\right)\left(w^{2}-1\right)}{\left(1-c_{0}\right)^{2} \bar{\lambda}^{2} w} \sin \left(\varphi_{0}\right) \sin \left(\varphi_{0}-2 \bar{\theta}\right)-\frac{2 c_{0}\left(1+\bar{\lambda}^{2}\right)\left(1+w^{2}\right)}{\left(1-c_{0}\right)^{2} \bar{\lambda} w} \cos \left(\varphi_{0}\right) .
\end{aligned}
$$


It then follows from expression (52) that the zeroth-order (relative) in-plane rotation, $\varphi_{0}$, can be shown to satisfy the identity

$$
l_{3}=\frac{g^{\prime}\left(\hat{I}_{0}^{(1)}\right)\left(1+\bar{\lambda}^{2}\right)}{w \bar{\lambda}^{2}\left(1-c_{0}\right)}\left[2 \bar{\lambda}\left(1+w^{2}\right) \sin \varphi_{0}-\left(\bar{\lambda}^{2}-1\right)\left(w^{2}-1\right) \sin \left(2 \varphi_{0}-2 \bar{\theta}\right)\right],
$$

where the definition

$$
l_{3}:=\left(M_{12}^{(2)}-M_{21}^{(2)}\right) \cos \bar{\psi}_{0}^{(2)}+\left(M_{11}^{(2)}+M_{22}^{(2)}\right) \sin \bar{\psi}_{0}^{(2)}
$$

has been used for conciseness. In this expression, $\bar{\psi}_{0}^{(2)}$ denotes the zeroth-order term in the expansion of the average in-plane rotation of the fibers in the small parameter $\delta$, such that $\varphi_{0}=\bar{\psi}_{0}^{(2)}-\bar{\psi}$. On the other hand, $l_{3}$ can be shown to correspond to the externally applied torque per unit volume of the fibers. This follows from the fact that $l_{3}=\rho^{0} c_{3}^{0}$, where $\rho^{0}$ is the density in the fibers and $c_{3}^{0}$ is the component of the body couple in the $X_{3}$ direction, as determined by expression (A.3) in Appendix A. Therefore, equation (74) provides a relation between the externally applied body couple and average in-plane rotation of fibers.

Substituting the leading-order terms for $\hat{\boldsymbol{F}}^{(1)}$ and $\overline{\boldsymbol{R}}^{(2)}$ into the expression (51), we obtain an estimate for the effective stored-energy function of the fiber-reinforced composites in the incompressibility limit (i.e., as $\kappa \rightarrow \infty$ or $\delta \rightarrow 0)$. In particular, it can be shown that the second term on the right side of (51) vanishes identically ${ }^{1}$, and therefore, we have that

$$
\begin{aligned}
\hat{W}_{\boldsymbol{M}}\left(\overline{\boldsymbol{F}} ; \boldsymbol{M}^{(2)}\right)=\hat{W}_{\boldsymbol{M}}\left(\bar{\lambda}, \bar{\theta}, \bar{\psi} ; \boldsymbol{M}^{(2)}\right) & =\left(1-c_{0}\right) g\left(\hat{I}_{0}^{(1)}\right) \\
& +c_{0}\left(M_{11}^{(2)}+M_{22}^{(2)}\right) \cos \bar{\psi}_{0}^{(2)}-c_{0}\left(M_{12}^{(2)}-M_{21}^{(2)}\right) \sin \bar{\psi}_{0}^{(2)},
\end{aligned}
$$

where $\hat{I}_{0}^{(1)}$ and $\bar{\psi}_{0}^{(2)}$ are given by expressions (73) and (74), respectively. It is interesting to remark at this point that $\hat{W}_{\boldsymbol{M}}$ may be written more compactly as

$$
\hat{W}_{\boldsymbol{M}}\left(\bar{\lambda}, \bar{\theta}, \bar{\psi} ; \boldsymbol{M}^{(2)}\right)=\operatorname{stat}_{\bar{\psi}(2)}\left\{\left(1-c_{0}\right) g\left(\hat{I}^{(1)}\right)+c_{0}\left(M_{11}^{(2)}+M_{22}^{(2)}\right) \cos \bar{\psi}^{(2)}-c_{0}\left(M_{12}^{(2)}-M_{21}^{(2)}\right) \sin \bar{\psi}^{(2)}\right\},
$$

where $\hat{I}^{(1)}$ is obtained by replacing $\bar{\psi}_{0}^{(2)}$ by $\bar{\psi}^{(2)}$ in expression (73) for $\hat{I}_{0}^{(1)}$. This is because the stationary condition in (77) is precisely the torque-rotation (74). In other words, the stationary condition in (77) for the variable $\bar{\psi}^{(2)}$ provides the solution $\bar{\psi}_{0}^{(2)}$, as given by relation (74). It follows that the corresponding macroscopic stress-strain relation is given by

$$
\overline{\boldsymbol{S}}=\frac{\partial \hat{W}_{\boldsymbol{M}}}{\partial \overline{\boldsymbol{F}}}=\left(1-c_{0}\right) g^{\prime}\left(\hat{I}_{0}^{(1)}\right) \frac{\partial \hat{I}_{0}^{(1)}}{\partial \overline{\boldsymbol{F}}},
$$

\footnotetext{
${ }^{1}$ Using the definition $\mathcal{S}^{(1)}=\mathcal{S}^{(1)}(\overline{\boldsymbol{F}})$, the second term on the right side of (51) can be shown to be proportional to

$$
\begin{aligned}
\overline{\boldsymbol{F}} \cdot\left[\overline{\boldsymbol{F}}-c_{0} \overline{\boldsymbol{R}}^{(2)}-\left(1-c_{0}\right) \hat{\boldsymbol{F}}^{(1)}\right] & =\overline{\boldsymbol{\Lambda}} \cdot\left[\overline{\boldsymbol{\Lambda}}-c_{0} \overline{\boldsymbol{R}}^{T} \overline{\boldsymbol{R}}^{(2)}-\left(1-c_{0}\right)(\overline{\boldsymbol{\Lambda}}+\boldsymbol{Y})\right] \\
& =c_{0}\left(\bar{\lambda}^{2}+\bar{\lambda}^{-2}\right)-c_{0} \cos \varphi_{0}\left(\bar{\lambda}+\bar{\lambda}^{-1}\right)-\left(1-c_{0}\right)\left(\bar{\lambda} Y_{11}+\bar{\lambda}^{-1} Y_{22}\right)
\end{aligned}
$$

which can then be shown to be zero when $Y_{11}$ and $Y_{22}$ are substituted from $(72)_{1,2}$, to the zeroth order in the small parameter $\delta$. Note that in obtaining the first identity we made used of relations (62) and (68).
} 
where the derivative of $\hat{I}_{0}^{(1)}$ with respect to $\overline{\boldsymbol{F}}$ is taken with $\bar{\psi}_{0}^{(2)}$ held fixed. This follows by use of the chain rule and the fact that the derivative with respect to $\bar{\psi}^{(2)}$ of the terms in brackets in expression (77) vanishes (by the stationarity condition). The important conclusion from this result is that the macroscopic stresses in the composite depend on $\boldsymbol{M}^{(2)}$ only through the combination $l_{3}$, as defined by expression (75).

\subsection{Computation of $\hat{W}_{\boldsymbol{F}}\left(\overline{\boldsymbol{F}} ; \boldsymbol{R}^{(2)}\right)$ in the incompressibility limit}

Having computed the effective energy $\hat{W}_{\boldsymbol{M}}\left(\overline{\boldsymbol{F}} ; \boldsymbol{M}^{(2)}\right)$ for the prescribed eigenstress $\boldsymbol{M}^{(2)}$, we can compute the dual energy $\hat{W}_{\boldsymbol{F}}\left(\overline{\boldsymbol{F}} ; \boldsymbol{R}^{(2)}\right)$ by means of the Legendre transformation (12). Thus, for the special case of two-phase fiber-reinforced composites undergoing 2D plane strains we have that, in the incompressibility limit,

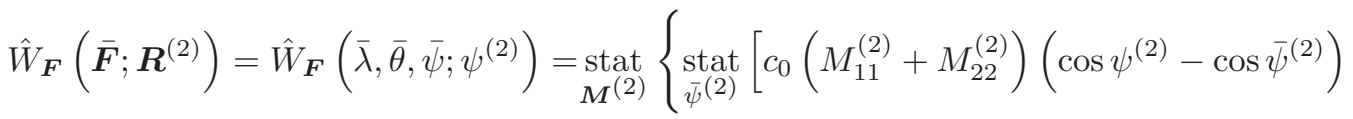

$$
\begin{aligned}
& \left.\left.-c_{0}\left(M_{12}^{(2)}-M_{21}^{(2)}\right)\left(\sin \psi^{(2)}-\sin \bar{\psi}^{(2)}\right)-\left(1-c_{0}\right) g\left(\hat{I}^{(1)}\right)\right]\right\}
\end{aligned}
$$

where $\psi^{(2)}$ denotes the prescribed average in-plane rotation of fibers. Now, interchanging the stationarity conditions in expression (79), we conclude that

$$
\bar{\psi}^{(2)}=\psi^{(2)},
$$

and therefore we obtain the result that

$$
\hat{W}_{\boldsymbol{F}}\left(\bar{\lambda}, \bar{\theta}, \bar{\psi} ; \psi^{(2)}\right)=-\left(1-c_{0}\right) g\left(\hat{I}^{(1)}\right)
$$

where $\hat{I}^{(1)}$ is now obtained by making the replacement $\bar{\psi}_{0}^{(2)}=\psi^{(2)}$ into the expression (73) for $\hat{I}_{0}^{(1)}$. It can then be seen that expressions (77) and (81) are consistent with the general duality expression (15). It is also important to emphasize that the final expression for $\hat{W}_{\boldsymbol{F}}\left(\overline{\boldsymbol{F}} ; \boldsymbol{R}^{(2)}\right)$, obtained above, is exactly identical to the corresponding expression in Lopez-Pamies and Ponte Castañeda (2006b), except for the sign and the fact that now the relative in-plane rotation of the fibers, $\varphi=\psi^{(2)}-\bar{\psi}$, is prescribed, and therefore need not satisfy the evolution equation (30) in Lopez-Pamies and Ponte Castañeda (2006b). Finally, it should also be noted that the torque-rotation relation (74) can be obtained by means of the identity (16) and expression (81) for $\hat{W}_{\boldsymbol{F}}\left(\overline{\boldsymbol{F}} ; \boldsymbol{R}^{(2)}\right)$. In particular, when the applied torque $l_{3}$ vanishes, we recover the result of Lopez-Pamies and Ponte Castañeda (2006b) for the particle rotation, namely, equation (74) with $l_{3}=0$.

\subsection{Linearization of the effective stored-energy functions $\hat{W}_{\boldsymbol{M}}$ and $\hat{W}_{\boldsymbol{F}}$}

In this subsection we provide the corresponding expressions for the effective stored-energy functions, as well as for the torque-rotation response of fiber-reinforced composites in the limit of infinitesimal strains and rotations. In this limit we have that

$$
\overline{\boldsymbol{F}}=\boldsymbol{I}+\overline{\boldsymbol{\epsilon}}+\overline{\boldsymbol{\omega}}
$$


where $\overline{\boldsymbol{\epsilon}}$ and $\overline{\boldsymbol{\omega}}(|\overline{\boldsymbol{\epsilon}}| \ll 1,|\overline{\boldsymbol{\omega}}| \ll 1)$ are the macroscopic infinitesimal strain and rotation tensors, respectively. For the special case of incompressible composites undergoing plane strains in the $X_{1}-X_{2}$ plane transverse to the fibers, the infinitesimal strain and rotation tensors can be written as

$$
[\overline{\boldsymbol{\omega}}]=\left[\begin{array}{cc}
0 & -\bar{\psi} \\
\bar{\psi} & 0
\end{array}\right], \quad \text { and } \quad[\overline{\boldsymbol{\epsilon}}]=\left[\begin{array}{cc}
\cos \bar{\theta} & -\sin \bar{\theta} \\
\sin \bar{\theta} & \cos \bar{\theta}
\end{array}\right]\left[\begin{array}{cc}
\bar{\epsilon} & 0 \\
0 & -\bar{\epsilon}
\end{array}\right]\left[\begin{array}{cc}
\cos \bar{\theta} & -\sin \bar{\theta} \\
\sin \bar{\theta} & \cos \bar{\theta}
\end{array}\right]^{T} .
$$

Thus, the effective energies and the torque-rotation relation in the infinitesimal limit can be simply obtained by letting $\bar{\lambda}=1+\bar{\epsilon}$ in the expressions of the previous subsections and assuming that $|\bar{\epsilon}| \ll 1,|\bar{\psi}| \ll 1$, and $\left|\bar{\psi}^{(2)}\right| \ll 1$. In particular, the torque-rotation relation (74) for prescribed torques reduces to

$$
\frac{l_{3}}{2 \mu}=\frac{1}{1-c_{0}}\left[\left(w+\frac{1}{w}\right) \varphi_{0}+\left(w-\frac{1}{w}\right) \bar{\epsilon} \sin (2 \bar{\theta})\right],
$$

while the corresponding effective stored-energy function (76) reduces to

$$
\hat{W}_{M}\left(\bar{\epsilon}, \bar{\theta}, \bar{\psi} ; l_{3}\right)=\frac{\mu}{1-c_{0}}\left[2 \bar{\epsilon}^{2}+c_{0}\left(w+\frac{1}{w}\right)\left(\bar{\epsilon}^{2}+\varphi_{0}^{2}\right)+2 c_{0}\left(w-\frac{1}{w}\right) \varphi_{0} \bar{\epsilon} \sin (2 \bar{\theta})\right]-c_{0} l_{3}\left(\varphi_{0}+\bar{\psi}\right),
$$

where $\varphi_{0}:=\bar{\psi}_{0}^{(2)}-\bar{\psi}$ denotes the relative in-plane rotation of the fibers as determined by the linearized torque-rotation relation (84).

Similarly, in the limit of infinitesimal deformations, the effective energy (81) for prescribed fiber rotations reduces to

$$
\hat{W}_{\boldsymbol{F}}\left(\bar{\epsilon}, \bar{\theta}, \bar{\psi} ; \psi^{(2)}\right)=-\frac{\mu}{1-c_{0}}\left[2 \bar{\epsilon}^{2}+c_{0}\left(w+\frac{1}{w}\right)\left(\bar{\epsilon}^{2}+\varphi^{2}\right)+2 c_{0}\left(w-\frac{1}{w}\right) \varphi \bar{\epsilon} \sin (2 \bar{\theta})\right],
$$

where $\varphi:=\bar{\psi}^{(2)}-\bar{\psi}$ denotes the prescribed infinitesimal rotation of the fibers relative to the macroscopic infinitesimal rotation $\bar{\psi}$.

\subsection{Comparisons with previously available results for the torque-rotation relation}

In this subsection we summarize previously available results for the relation between the torque and rotation of the fibers. In particular, we summarize the results for the single fiber problem in the limit of infinitesimal deformations, as well as results for the rotation of a rigid cylindrical fiber embedded in a shell of a hyperelastic material undergoing a finite twist.

The relation between the applied torque and rotation of a single rigid ellipsoidal inclusion embedded in an infinite linearly elastic medium has been previously obtained by Walpole (1991) (see also Siboni and Ponte Castañeda, 2012, for a more recent calculation, which also includes the effects of affine deformation on the boundary). In particular, for a single rigid inclusion with general ellipsoidal shape in an infinite medium under the affine displacement boundary condition

$$
\mathbf{u}(\mathbf{x})=\bar{\epsilon} \mathbf{x}+\bar{\omega} \mathbf{x} \quad \text { as } \quad|\mathbf{x}| \rightarrow \infty
$$

Siboni and Ponte Castañeda (2012) have obtained the relation

$$
l_{i}=\varepsilon_{i j k} \tau_{j k}, \quad \text { where } \quad \boldsymbol{\tau}=\left[\mathbb{S}-\mathbb{R P}^{-1} \mathbb{Q}\right]\left[\overline{\boldsymbol{\omega}}-\overline{\boldsymbol{\omega}}^{(2)}-\mathbb{R P}^{-1} \overline{\boldsymbol{\epsilon}}\right]
$$


between the externally applied torque (per unit volume) $\boldsymbol{l}$ and the infinitesimal rotation of the inclusion $\overline{\boldsymbol{\omega}}^{(2)}$ (for prescribed $\overline{\boldsymbol{\epsilon}}$ and $\overline{\boldsymbol{\omega}}$ on the boundary). In expression (88), the fourth-order tensors $\mathbb{P}, \mathbb{R}, \mathbb{Q}$, and $\mathbb{S}$ are microstructural (Eshelby-type) tensors that depend on the properties of the matrix material and shape of the inclusion (see Walpole, 1991; Siboni and Ponte Castañeda, 2012, for more details). For the special case of a long cylindrical fiber with shape tensor (56), embedded in an incompressible matrix with shear modulus $\mu$, and under the $2 \mathrm{D}$ plane strain conditions, equation (88) can be shown to reduce to

$$
\frac{l_{3}}{2 \mu}=\left(w+\frac{1}{w}\right)\left(\bar{\psi}^{(2)}-\bar{\psi}\right)+\left(w-\frac{1}{w}\right) \bar{\epsilon} \sin (2 \bar{\theta}),
$$

where $l_{3}$ is the externally applied torque (per unit volume of the fiber), $\bar{\psi}^{(2)}$ is the infinitesimal rotation (in the $X_{1}-X_{2}$ plane) of the fiber measured in the counterclockwise sense from the positive $X_{1}$ direction, and $\bar{\psi}, \bar{\epsilon}$, and $\bar{\theta}$ are the macroscopic loading parameters, as defined in (83). It can then be seen that our more general result (84) for infinitesimal deformations and fiber rotations reduces exactly to the expression (89) for a single elliptical fiber in the limit as $c_{0} \rightarrow 0$.

In the context of finite deformations, Kanner and Horgan (2008) provided an exact analytical solution for the azimuthal shearing of hollow elastic cylinders of various types of nonlinear hyperelastic materials, generalizing the classical solution of Rivlin (1949) for a neo-Hookean cylindrical shell. In particular, the solution of Kanner and Horgan (2008) can be used to obtain the relation between torque and rotation of a rigid cylinder with radius $A$, enclosed by a hyperelastic shell with (outer) radius $B>A$, for a Gent elastomer matrix with constitutive law of the form (59), yielding the expression

$$
\bar{\psi}^{(2)}=\frac{\mu J_{m}}{2 l_{3} \hat{c}_{0}}\left\{\sqrt{1+\hat{c}_{0}^{2} \alpha}-\hat{c}_{0} \sqrt{1+\alpha}-\hat{c}_{0} \sqrt{\alpha} \ln \left[\frac{\hat{c}_{0} \sqrt{\alpha}+\sqrt{1+\hat{c}_{0}^{2} \alpha}}{\sqrt{\alpha}+\sqrt{1+\alpha}}\right]-\left(1-\hat{c}_{0}\right)\right\}
$$

for the rotation of the inner cylinder when the outer boundary of the elastic shell is held fixed. In this last expression, the definitions

$$
\alpha:=\frac{l_{3}^{2}}{J_{m} \mu^{2}} \quad \text { and } \quad \hat{c}_{0}:=\frac{A^{2}}{B^{2}}
$$

are used for conciseness. Note that expression (90) can be used as an approximate expression for the torquerotation response of a distribution of circular fibers in a Gent elastomer at zero macroscopically imposed deformation, where $\hat{c}_{0}$, as defined above, can be thought of as a measure for the fiber volume fraction. In the limit as $\hat{c}_{0} \rightarrow 0$, the above result reduces to

$$
\bar{\psi}^{(2)}=\frac{\mu J_{m}}{2 l_{3}}\{\sqrt{\alpha} \ln [\sqrt{\alpha}+\sqrt{1+\alpha}]-[\sqrt{1+\alpha}-1]\},
$$

corresponding to the case of a single cylindrical fiber in an infinite Gent elastomer. Finally, in the limit as $J_{m} \rightarrow \infty$ (i.e., for Neo-Hookean materials), expressions (90) and (92) respectively reduce to

$$
l_{3}=\frac{4 \mu}{\left(1-c_{0}\right)} \bar{\psi}^{(2)} \quad \text { and } \quad l_{3}=4 \mu \bar{\psi}^{(2)} .
$$

Interestingly, this last result for the single fiber agrees exactly with the corresponding linear solution (89), for circular cross-sections $(w=1)$, and no deformation on the external boundary $(\bar{\epsilon}=\bar{\psi}=0)$, even for large 
rotations. On the other hand, our more general expression (74), when specialized for a neo-Hookean matrix and circular fibers $(w=1)$, as well as fixed deformation on the external boundary $(\bar{\lambda}=1)$, reduces to

$$
l_{3}=\frac{4 \mu}{\left(1-c_{0}\right)} \sin \bar{\psi}^{(2)} \quad \text { and } \quad l_{3}=4 \mu \sin \bar{\psi}^{(2)}
$$

for non-dilute and dilute concentrations, respectively. It can thus be seen that, in particular, the dilute result $(94)_{2}$ agrees with the exact expression (92) for a neo-Hookean matrix to second order in $\bar{\psi}^{(2)}<<1$. Note that our dilute result $(94)_{2}$ also holds for Gent-type materials, and is thus insensitive to the value of $J_{m}$.

\section{Discussion of the results}

We begin the discussion of the results of this paper by making a few more detailed comparisons with the known exact results mentioned at the end of the previous section. Thus, Fig. 2 shows comparisons for the torque-rotation relation $(74)$ for cylindrical fibers $(w=1)$ and no macroscopic deformation $(\bar{\lambda}=1)$. In particular, Fig. 2(a) provides comparisons of our estimate $(94)_{2}$ for a single fiber $\left(c_{0} \rightarrow 0\right)$ with the corresponding result (92) of Kanner and Horgan (2008) for Gent materials with several values of $J_{m}$, including Rivlin's solution for the neo-Hookean material $\left(J_{m} \rightarrow \infty\right)$. As already mentioned, our estimate is exact for small rotations, but is insensitive to the value of $J_{m}$, and actually underestimates the value of the torque, even for the neo-Hookean case, at finite values of the rotation. The error can be seen to be roughly $9 \%$ for neo-Hookean materials and $15 \%$ for Gent materials with $J_{m}=10$ at a rotation $\bar{\psi}^{(2)}$ of $\pi / 4$, while it is less than 4 and $8 \%$ at $\bar{\psi}^{(2)}=\pi / 6$. Figure 2 (b) shows the comparison between the homogenization estimate (74) and the nonlinear result (90) of Kanner and Horgan (2008) for a fixed concentration of the fibers $\left(c_{0}=\hat{c}_{0}=0.4\right)$. In this connection, it should be emphasized that the results of Kanner and Horgan (2008) do not necessarily correspond to an actual composite (since they correspond to a single fiber), but may still account approximately for fiber interactions through the reduction of the matrix phase in the composite cylinder. It is observed that our estimates still agree exactly with the results of Kanner and Horgan (2008) for small rotations, but are now able to capture the stiffening effect due to the chain extensibility parameter $J_{m}$ in the Gent model. However, our predictions are more compliant than the corresponding results of Kanner and Horgan (2008) for given values of $J_{m}$, consistent with the earlier observations for dilute concentrations of fibers. Interestingly, the results show that our estimates can be improved relative to the exact results by making use of an artificially higher value of $J_{m}$ in order to compensate for the errors in the predictions. For example, our results for $J_{m}=10$ are fairly close to the "exact" results for $J_{m}=20$.

It is important at this stage to comment briefly on the potential source of the errors associated with the new homogenization estimates, as well as on the overall range of validity of such estimates. In this context, it is relevant to recall the simplifying assumption in the work of Lopez-Pamies and Ponte Castañeda (2006b) that the elasticity tensor $\mathbb{L}^{(1)}$ of the matrix of the LCC was taken to have orthotropic symmetry, as given by (66). While this assumption may be expected to be reasonably accurate for problems with prescribed macroscopic deformations and no torques (or rotations) on the fibers, such symmetry assumptions for $\mathbb{L}^{(1)}$ 


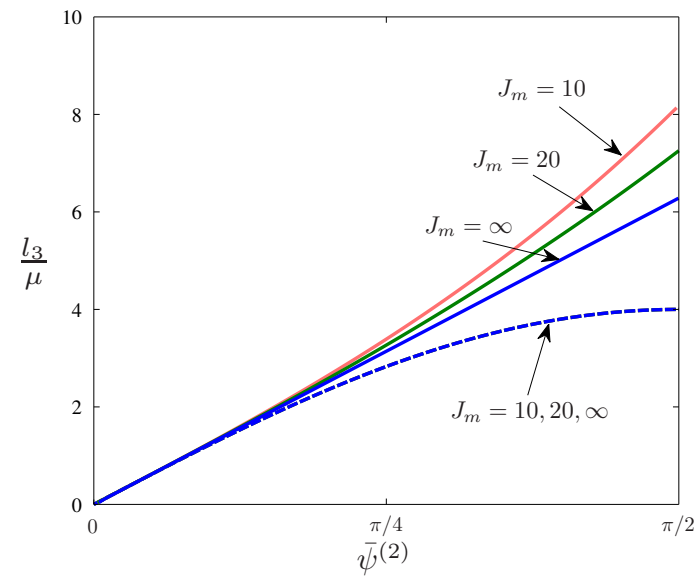

(a)

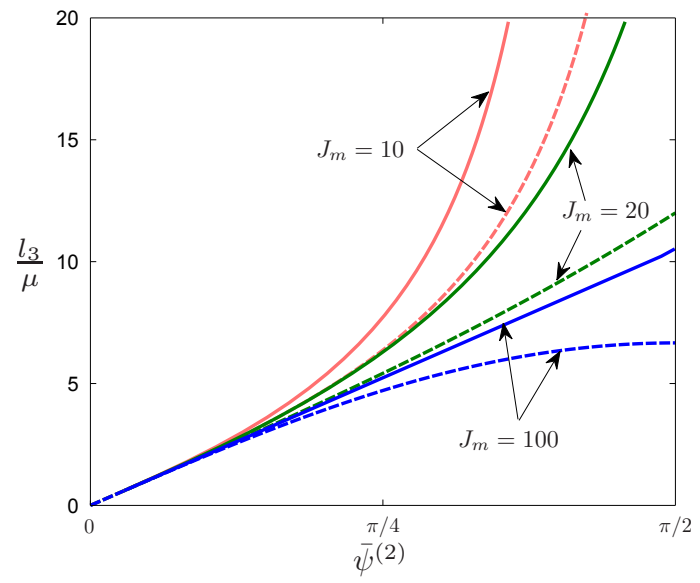

(b)

Figure 2: Torque-rotation curves for a dispersion of rigid fibers of circular cross section $(w=1)$ in an Gent elastomer under zero macroscopic deformation. (a) Comparison of the new estimate $(94)_{2}$ for a single fiber $\left(c_{0} \rightarrow 0\right)$ with the corresponding result (92) of Kanner and Horgan (2008) for Gent materials with several values of $J_{m}$, including Rivlin's solution for the neo-Hookean material $\left(J_{m} \rightarrow \infty\right)$. (b) Comparison of the new estimate with the nonlinear results of Kanner and Horgan (2008) for a fixed concentration $\left(c_{0}=\hat{c}_{0}=0.4\right)$ of circular fibers in a Gent elastomer. The solid lines correspond to the nonlinear results of (90), while the dashed lines to relation (74).

may no longer be appropriate for situations where the inclusions are also subjected to couples, especially when large rotations are involved. In any event, the previous comparisons for cylindrical fibers and vanishing external loads have allowed us to generate a rough estimate for the range of validity of our results for general particle shapes and more general loading conditions. Thus, we have seen that the errors should be less than $15 \%$ for $\bar{\psi}^{(2)}=\pi / 4$ and $8 \%$ for $\bar{\psi}^{(2)}=\pi / 6$. For this reason, we will restrict our attention henceforth to particle rotations of less than $\pi / 4$. While such fiber rotations are not very large, they are well beyond the expected linear regime and large enough to be able to take into account the effect of the additional magnetically or electrically driven rotations in the MAE and DEC material systems, which were used in the introduction to motivate this work.

Next, we investigate the effect of different microstructural parameters (strain-locking parameter $J_{m}$, concentration $c_{0}$, and aspect ratio $w$ ) on the torque-rotation response curves at zero macroscopic deformations. Figure 3 shows the effect of the Gent strain-locking parameter $J_{m}$ on the torque-rotation relation for fibers of elliptical cross section $(w=2)$, when no macroscopic deformation is applied on the boundary (i.e., $\bar{\lambda}=1)$. In particular, Fig. 3(a) shows the results for $c_{0}=0.25$, while Fig. 3(b) shows the results for $c_{0}=0.5$. Consistent with earlier observations in the context of Fig. 2, it is seen that the parameter $J_{m}$ has a significantly stronger stiffening effect at the higher fiber volume fraction. This can be attributed to the stronger interactions between the fibers at the larger volume fractions. However, as also pointed out earlier, the slight softening effect observed for the neo-Hookean elastomer at the larger values of $\bar{\psi}^{(2)}$ is expected to be a consequence of errors in the homogenization estimates associated with the use of simplified linearization 


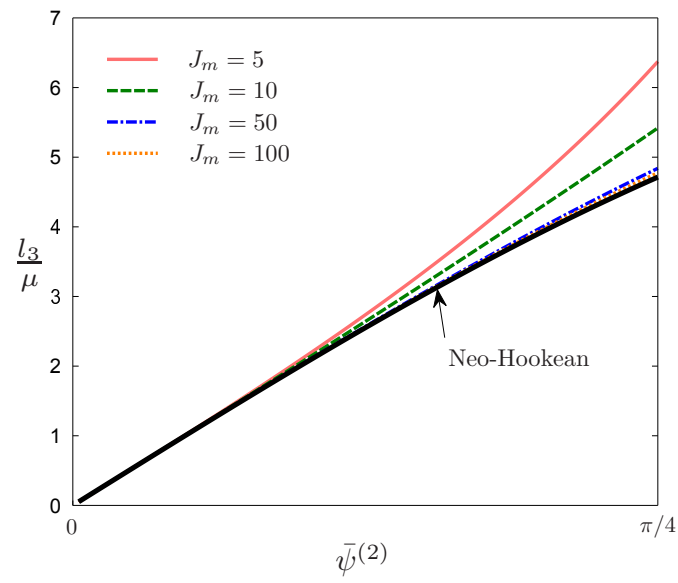

(a)

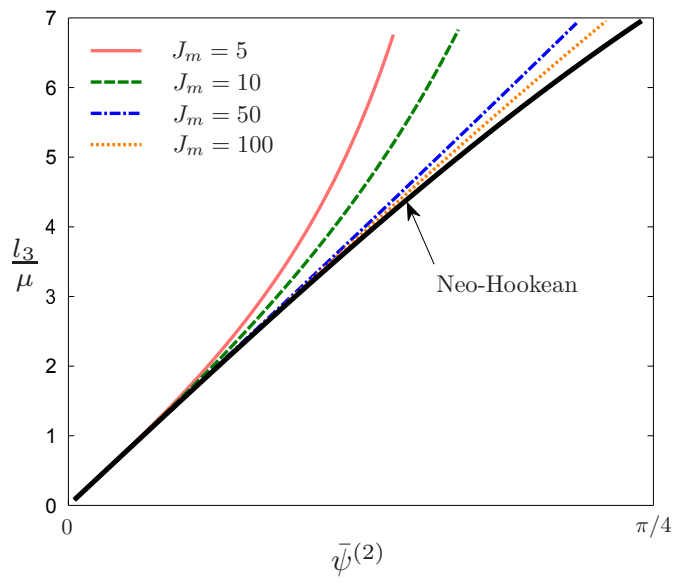

(b)

Figure 3: Results for the behavior of the external torque $l_{3}$ as a function of the in-plane rotation $\bar{\psi}^{(2)}$ for different values of the strain-locking parameter $J_{m}$. (a) $c_{0}=0.25$, and (b) $c_{0}=0.5$. In this figure $w=2, \bar{\lambda}=1$ and $\bar{\psi}=0$.

schemes.

Figure 4 shows the effect of the fiber volume fraction on the torque-rotation curves when no deformation is applied on the boundary $(\bar{\lambda}=1)$. In particular, Fig. 4(a) shows the results for circular $(w=1)$ fibers in a Gent elastomer with $J_{m}=100$, while Fig. 4(b) shows the corresponding results for $J_{m}=10$. As can be seen from these figures, the torque (per unit volume) required to achieve a certain rotation increases with increasing fiber volume fraction, which can be easily understood by recalling that the presence of rigid fibers

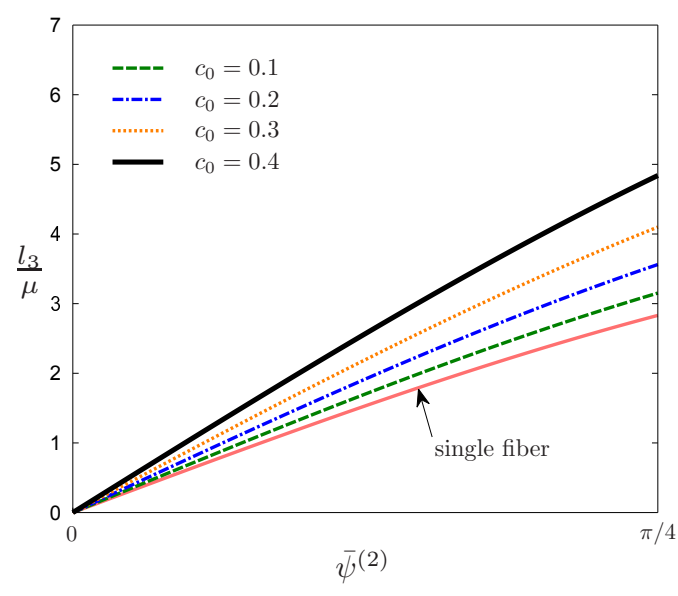

(a)

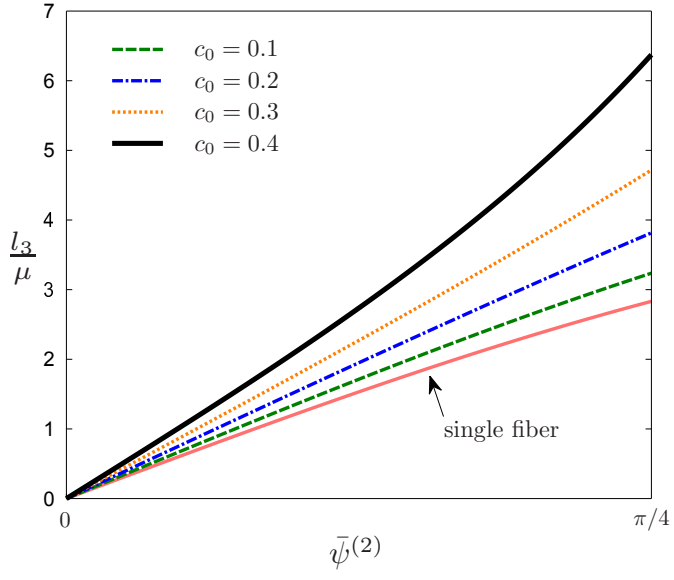

(b)

Figure 4: Results for the external torque $l_{3}$ as a function of the in-plane rotation $\bar{\psi}(2)$ for different values of the fiber concentration. (a) $J_{m}=100$, and (b) $J_{m}=10$. In this figure $w=1, \bar{\lambda}=1$ and $\bar{\psi}=0$.

makes the composite effectively stiffer. As also expected, the stiffening effect is more marked for the smaller value of the strain-locking parameter $\left(J_{m}=10\right)$ than for the larger value $\left(J_{m}=100\right)$. In addition, it should 
be noted that - unlike the case for $J_{m}$ - the value of the fiber volume fraction has a significant effect on the torque-rotation curves even for small rotations.

Figure 5 shows the effect of the aspect ratio on the torque-rotation response curves. In particular Fig.

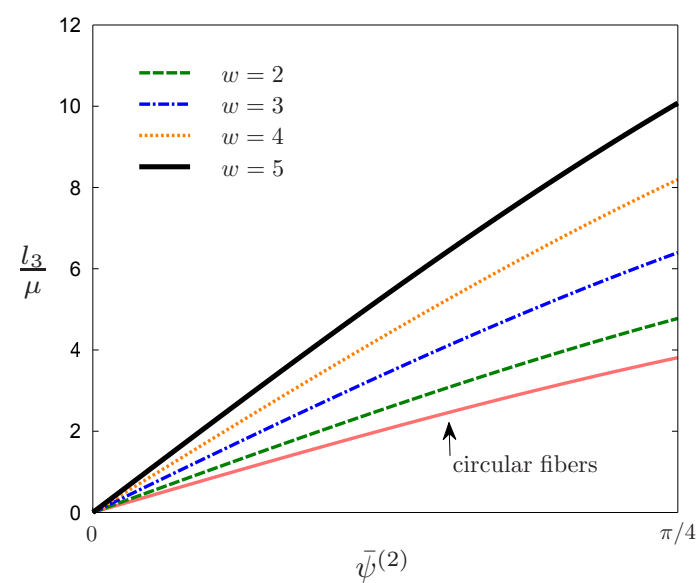

(a)

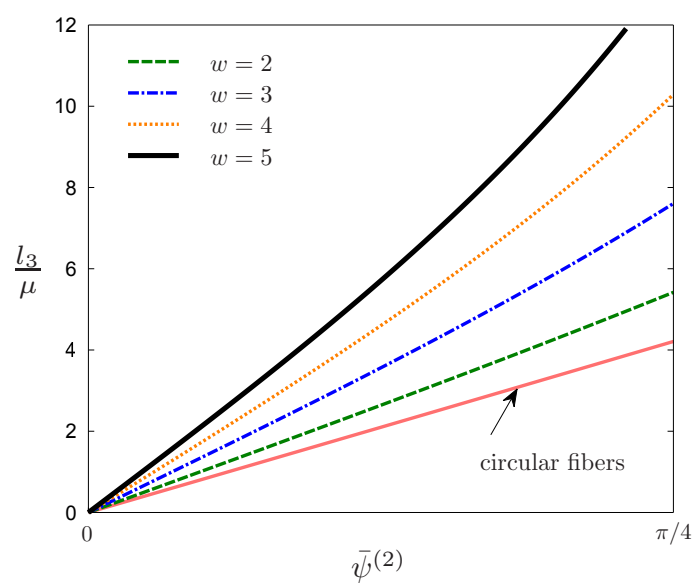

(b)

Figure 5: Results for the external torque $l_{3}$ as a function of the in-plane rotation $\bar{\psi}^{(2)}$ for different values of the aspect ratio. (a) $J_{m}=100$, and (b) $J_{m}=10$. In this figure $c_{0}=0.25, \bar{\lambda}=1$ and $\bar{\psi}=0$.

5(a) shows the results for $J_{m}=100$ and Fig. 5(b) shows the corresponding results for $J_{m}=10$. As can be seen from these figures, larger values of the aspect ratio of the fibers results in stiffer torque-rotation responses, while the effect is stronger for the smaller value of $J_{m}$. It should be emphasized that - as was the case for the volume fraction, but not for $J_{m}$ - the value of the fiber aspect ratio has a significant effect on the torque-rotation curves even for small rotations.

Next, we consider the effect of the macroscopic deformation, as determined by the loading parameters $\bar{\lambda}, \bar{\theta}$, and $\bar{\psi}$. Thus, Fig. 6 shows the effect of the macroscopic pure shear deformations of the form $\overline{\boldsymbol{F}}=$ $\bar{\lambda} \hat{\mathbf{E}}_{1} \otimes \hat{\mathbf{E}}_{1}+\bar{\lambda}^{-1} \hat{\mathbf{E}}_{2} \otimes \hat{\mathbf{E}}_{2}$ on the torque-rotation curves. (Recall that the long axis of the fibers is aligned with the $\mathbf{E}_{2}$ direction.) In particular, Fig. 6(a) shows the results for $J_{m}=100$, while Fig. 6(b) shows the results for $J_{m}=10$. As can be seen from these figures, when $\bar{\lambda}<1$ (i.e., compression in the $X_{1}$ direction and elongation in the $X_{2}$ direction), the torque-rotation response is generally stiffer than for the no deformation case. This is easily explained by noting that stretching the composite along the longer in-plane axis of the fibers ( $X_{2}$ direction for the specific example discussed here) tends to keep the fibers aligned with the $X_{2}$ direction, thus opposing the imposed rotation on the fibers, and requiring a larger torque to produce the same fiber rotation. On the other hand, when $\bar{\lambda}>1$ (i.e., elongation in the $X_{1}$ direction and compression in the $X_{2}$ direction) the torque-rotation response is generally softer compared to the no deformation case. This is due to the fact that the fibers tend to rotate away from the compressive axis when the material is stretched perpendicular to the larger in-plane axis of the fibers. In this case, the external deformation tends to enhance the fiber rotations, thus requiring a lower torque to produce the same overall rotation as the no 


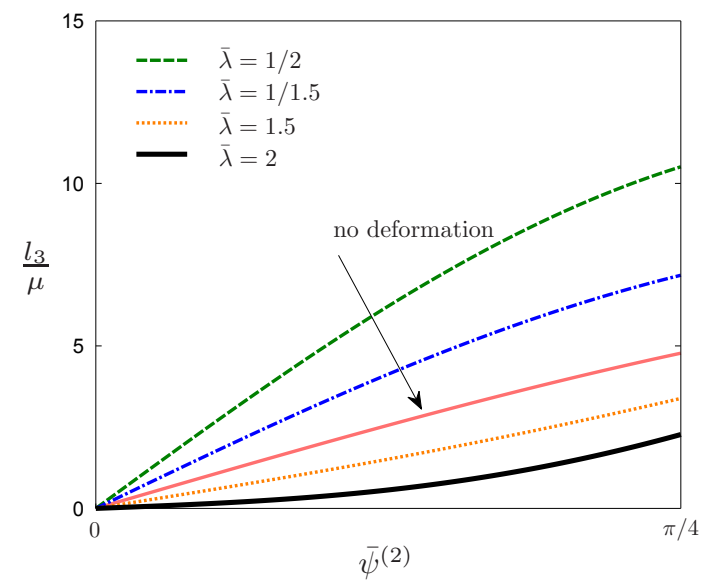

(a)

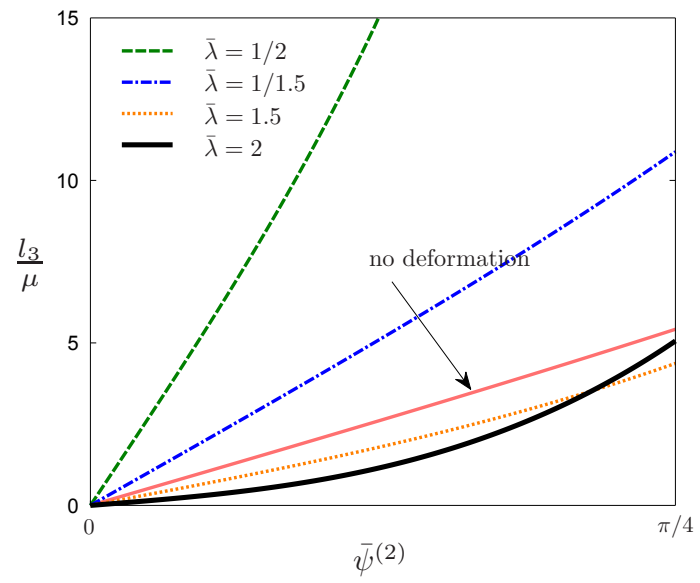

(b)

Figure 6: Results for the external torque $l_{3}$ as a function of the in-plane rotation $\bar{\psi}^{(2)}$ for different values of the macroscopic pure shear $\bar{\lambda}$, such that $\overline{\boldsymbol{F}}=\bar{\lambda} \hat{\mathbf{E}}_{1} \otimes \hat{\mathbf{E}}_{1}+\bar{\lambda}^{-1} \hat{\mathbf{E}}_{2} \otimes \hat{\mathbf{E}}_{2}$. (a) $J_{m}=100$, and (b) $J_{m}=10$. In this figure $c_{0}=0.25, w=2, \bar{\theta}=0$, and $\bar{\psi}=0$.

external stretch case. Note that for large enough stretches (e.g., $\bar{\lambda}=2$ in the specific example here) the slope of the torque-rotation curve at $\bar{\psi}^{(2)}=0$ tends to zero. In other words, a very small external torque will result in large rotations of the fibers. The point at which the slope of the torque-rotation curve vanishes would be expected to correspond to the onset of an instability and can in fact be shown to coincide with loss of strong ellipticity of the effective energy of the composite, as previously discussed by Lopez-Pamies and Ponte Castañeda (2006b).

Figure 7 shows the effect of a macroscopic simple shear of the form $\overline{\boldsymbol{F}}=\boldsymbol{I}+\bar{\gamma} \hat{\mathbf{E}}_{1} \otimes \hat{\mathbf{E}}_{2}$ on the torquerotation response curves. In particular, Fig. 7 (a) shows the results for $J_{m}=100$, while Fig. 7 (b) shows the results for $J_{m}=10$. As can be seen from these figures, the presence of a non-zero shear (i.e., $\bar{\gamma} \neq 0$ ) produces a shift of the torque-rotation curves away from the origin. This is easily understood because the shear produces a rotation of the fibers (positive, or counter-clockwise, for $\bar{\gamma}<0$, and negative for $\bar{\gamma}>0$ ). However, it is also seen that the slope of the torque-rotation curves at the horizontal intercepts increases with the magnitude of the macroscopic shear, which means that the shift in the curves is non-uniform. This has the interesting effect that the curves for different amounts of shear can intersect each other for appropriate values of the fiber rotations. In other words, different combinations of the applied fiber torques and externally applied shear can lead to the same overall rotation. Finally, it is noted that the stiffening effect of the applied shear is again significantly more pronounced for smaller values of $J_{m}$.

Thus far, we have studied the effect of different microstructural parameters as well as the macroscopic deformation on the relation between the externally applied torques and the rotation of the fibers in fiberreinforced composites. Next, we investigate the effect of the externally applied torques on the macroscopic 


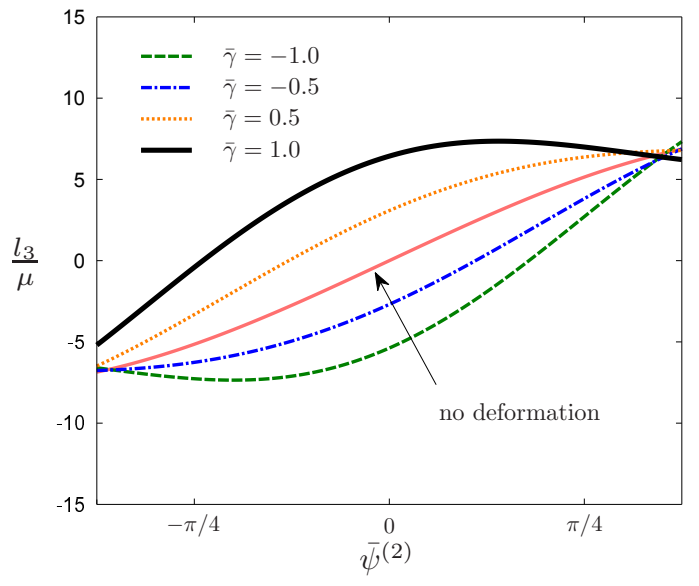

(a)

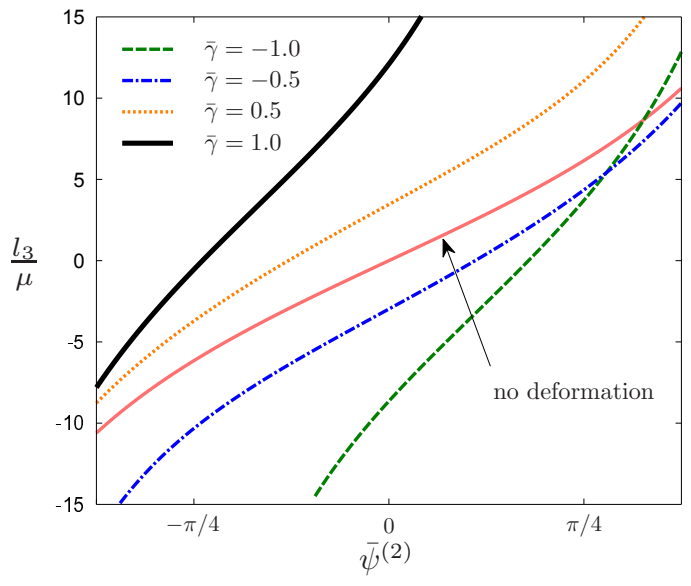

(b)

Figure 7: This figure shows the behavior of the external torque $l_{3}$ as a function of the in-plane rotation $\bar{\psi}^{(2)}$ for different values of the macroscopic simple shear $\bar{\gamma}$, such that $\overline{\boldsymbol{F}}=\boldsymbol{I}+\bar{\gamma} \hat{\mathbf{E}}_{1} \otimes \hat{\mathbf{E}}_{2}$. (a) shows the results for $J_{m}=100$, and (b) shows the results for $J_{m}=10$. In this figure $c_{0}=0.3$ and $w=2$.

response of such composites as determined by the effective constitutive response (c.f., $(17)_{1}$ )

$$
\overline{\boldsymbol{T}}=\bar{J} \frac{\partial \hat{W}_{\boldsymbol{M}}}{\partial \overline{\boldsymbol{F}}} \overline{\boldsymbol{F}}^{T}-p \boldsymbol{I}
$$

In particular, we consider the special case where the deformation is aligned with the laboratory axes (see Fig. 1) and of the form

$$
\overline{\boldsymbol{F}}=\bar{\lambda} \hat{\mathbf{E}}_{1} \otimes \hat{\mathbf{E}}_{1}+\bar{\lambda}^{-1} \hat{\mathbf{E}}_{2} \otimes \hat{\mathbf{E}}_{2}
$$

Note that this means that the composite is being compressed along the long axis of the fibers. For such deformations the relevant components of the macroscopic stress are obtained as follows

$$
\begin{aligned}
\bar{T}_{11}-\bar{T}_{22} & =\bar{\lambda} \frac{\partial \hat{W}_{M}}{\partial \bar{\lambda}} \\
\bar{T}_{12} & =\frac{\bar{\lambda}^{2}}{\bar{\lambda}^{4}-1} \times \frac{\partial \hat{W}_{M}}{\partial \bar{\theta}}-\frac{1}{\bar{\lambda}^{2}+1} \times \frac{\partial \hat{W}_{M}}{\partial \bar{\psi}} \\
\bar{T}_{21} & =\frac{\bar{\lambda}^{2}}{\bar{\lambda}^{4}-1} \times \frac{\partial \hat{W}_{M}}{\partial \bar{\theta}}+\frac{\bar{\lambda}^{2}}{\bar{\lambda}^{2}+1} \times \frac{\partial \hat{W}_{M}}{\partial \bar{\psi}}
\end{aligned}
$$

As can be seen in the above equations, the macroscopic Cauchy stress in the presence of external torques is no longer symmetric. In fact, using $(97)_{2,3}$ it can be shown that

$$
\bar{T}_{12}-\bar{T}_{21}=-\frac{\partial \hat{W}_{M}}{\partial \bar{\psi}}=c_{0} l_{3}
$$

where in obtaining the second identity we made use of equation (76) and the identity (75). Note that this is consistent with the general constitutive relations discussed in Appendix A, for homogeneous materials in the presence of external body couples (see equation (A.3) $)_{2}$ and the discussions associated with it). 
Figure 8 shows the effect of the loading parameter $\bar{\lambda}$ under the uniform deformation (96) on the normal and shear components of the macroscopic stress $\bar{T}_{i j}$ and particle rotation $\bar{\psi}^{(2)}$, for a fixed value of the aspect ratio of the fibers $(w=2)$ and different values of the normalized particle torques $l_{3} / \mu$. As can be seen from Fig. 8(a), the difference in the normal components of the macroscopic Cauchy stress, $\bar{T}_{11}-\bar{T}_{22}$ (i.e., the relevant stress measure in the incompressibility limit) changes slightly as the magnitude of the external torque increases. In fact, according to Fig. 8(a), the response of the composite becomes slightly softer at

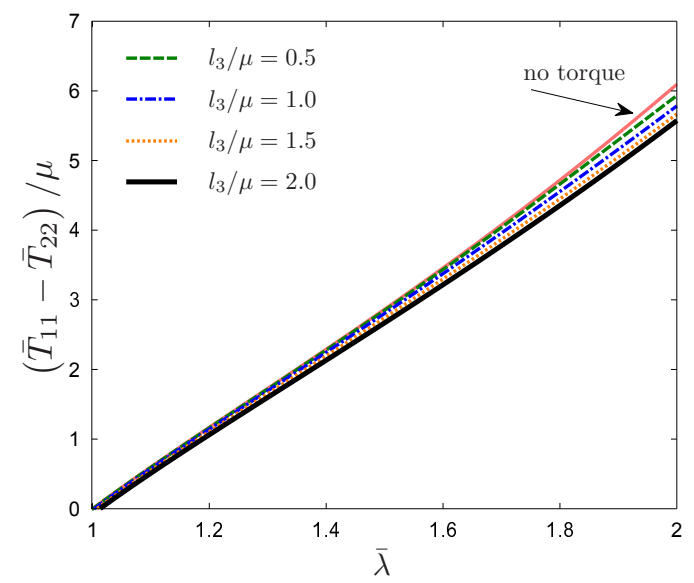

(a)

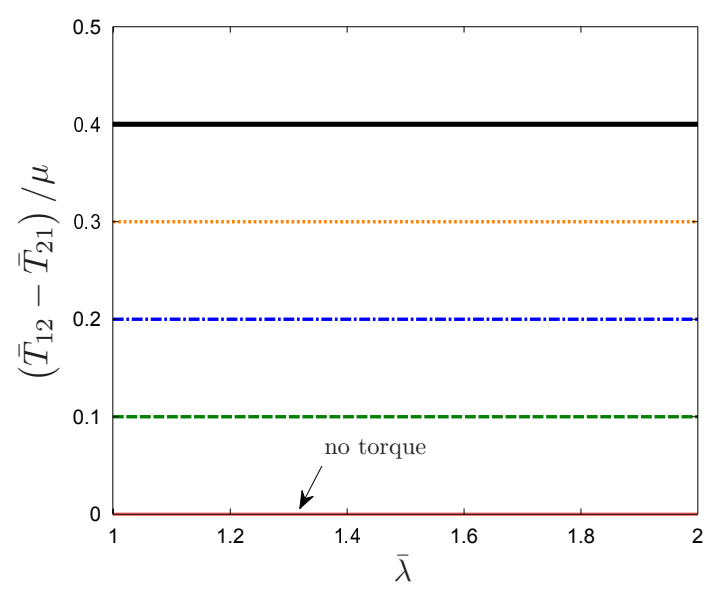

(c)

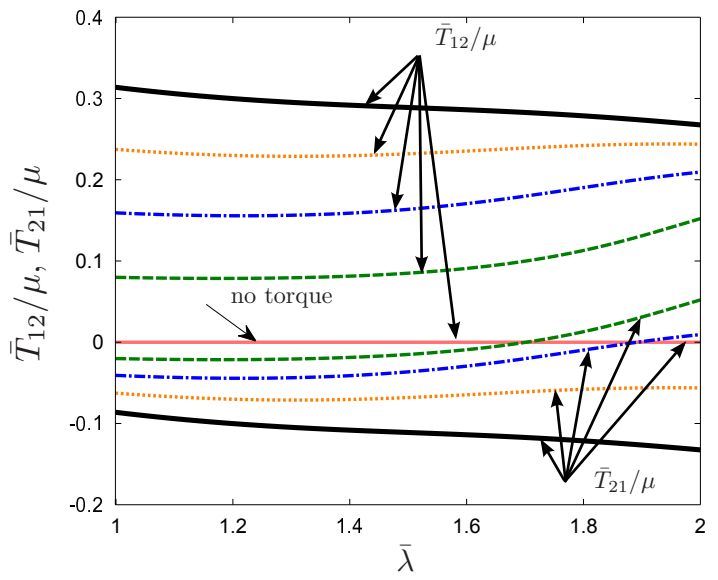

(b)

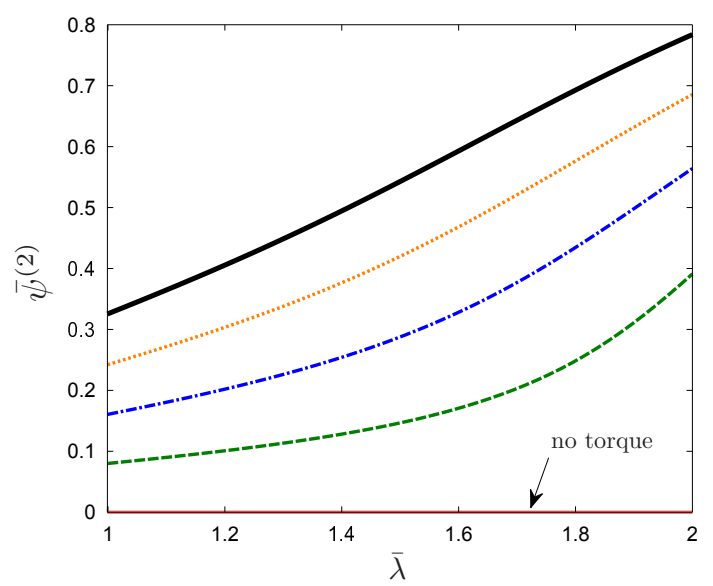

(d)

Figure 8: This figure shows the effect of the externally applied torques on the stress-stretch response of the composite under axis-aligned pure shear deformations of the form (96), as obtained by (a) shows $\bar{T}_{11}-\bar{T}_{22}$, (b) $\bar{T}_{12}$ and $\bar{T}_{21}$, (c) $\bar{T}_{12}-\bar{T}_{21}$, and (d) $\bar{\psi}^{(2)}$. In this figure $c_{0}=0.2, w=2, J_{m}=\infty$, and $\bar{\theta}=0$.

larger deformations in the presence of external couples. This is due to the fact that external torques cause the fibers to rotate away from their aligned (with the coordinate axes) orientations, and this is known to make the composite effectively softer (e.g., see Lopez-Pamies and Ponte Castañeda, 2006b). On the other hand, as can be seen in Fig. 8(b), the shear components of the macroscopic stress have a stronger dependence -in 
relative terms - on the magnitude of the applied torque. Note that, as shown in Fig. 8(c) and consistent with expression (98), the difference $\bar{T}_{12}-\bar{T}_{21}$ becomes identically zero in the absence of external torques, and is exactly equal to the net external torque per unit volume of the composite (i.e., $c_{0} l_{3}$ ) otherwise. For completeness, Fig. 8(d) shows the evolution of the average particle rotation $\bar{\psi}^{(2)}$ as a function of the applied stretch $\bar{\lambda}$ for the various values of the applied particle torques. Clearly, the additional torques facilitate the rotation of the fibers, since they break the symmetry of the aligned loading conditions.

Finally, Figure 9 shows the corresponding results for the normal and shear components of the macroscopic stress $\bar{T}_{i j}$ and particle rotation $\bar{\psi}^{(2)}$, for a fixed value of the particle torques $\left(l_{3} / \mu=2\right)$ and different values of the aspect ratio of the fibers $w$. As can be seen from Fig. 9(a), the difference in the normal components of

the macroscopic stress, $\bar{T}_{11}-\bar{T}_{22}$ changes only slightly, becoming softer, as the fiber aspect ratio $w$ increases. On the other hand, as can be seen in Fig. 9(b), the aspect ratio has a stronger effect - in relative terms - on the shear components of the macroscopic stress. Also, as can be seen in Fig. 9(c), the difference $\bar{T}_{12}-\bar{T}_{21}$ is the same for all values of $w$, since the external couple is the same for all the cases. Another point worth mentioning is the difference between the curve for circular fibers (i.e., $w=1$ ) and elliptical fibers (i.e., $w>1$ ) in Fig. 9(d). As can be seen in this figure the in-plane rotation of circular fibers under fixed external torques decreases with increasing $\bar{\lambda}$. This is easily explained by recalling that at larger deformations the composite becomes effectively stiffer. On the other hand, the in-plane rotation of elliptical fibers increases as the deformations progress. This is due to the tendency of fibers to align their larger in-plane axis with direction of the maximum principal stretch ( $X_{1}$ axis, for the specific example shown in this figure). Note that the overall stiffening behavior of the composites consisting of elliptical fibers as the deformation progresses is manifested here by the reduction in the slope of the rotations-stretch curves in Fig. 9(d).

\section{Concluding Remarks}

In this paper we have addressed the problem of determining the macroscopic response of elastomers that are reinforced by aligned fibers of elliptical cross section, and subjected to combined deformation on the external boundary and prescribed torques or rotations on the internal fibers. For this purpose, we have recognized that prescribed torques can be thought of as eigenstresses, and made use of the variational formulation (9) for a modified elasticity homogenization problem with prescribed eigenstresses leading to expression (11) for the associated rotations. Furthermore, this problem was shown to be equivalent to the problem (13) with prescribed average deformation gradients, via the Legendre transformation (12), leading to the inverse relation (16) for the eigenstresses as functions of the average rotations. The resulting constitutive model for the fiber-reinforced composite accounts for finite strains and rotations, and includes an evolution equation for the (in-plane) rotation of the fibers in response to the applied torques. The main results for the case of externally applied torques are provided by equations (74) and (76) for the particle rotation and effective stored-energy function, respectively. On the other hand, the main result for the case where the (inplane) average rotation of the fibers is prescribed is given by equation (81). The resulting constitutive model 


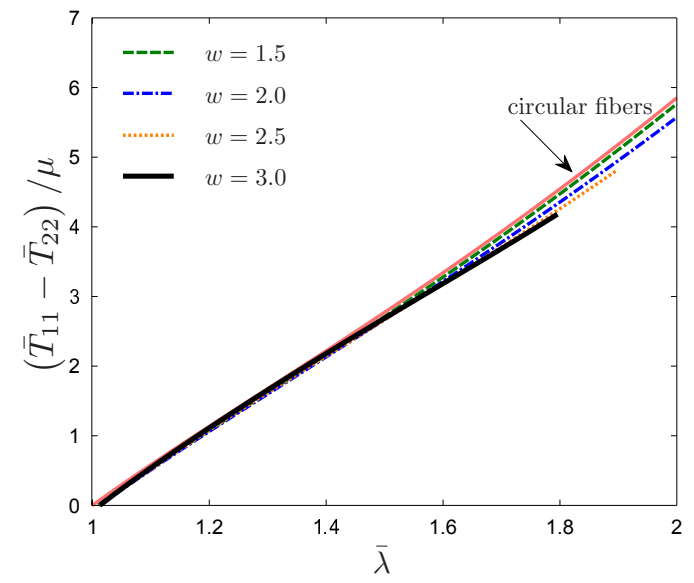

(a)

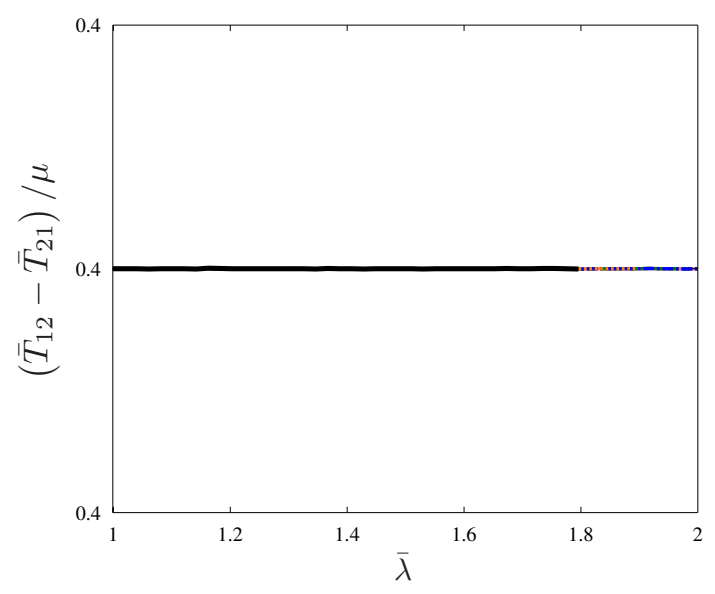

(c)

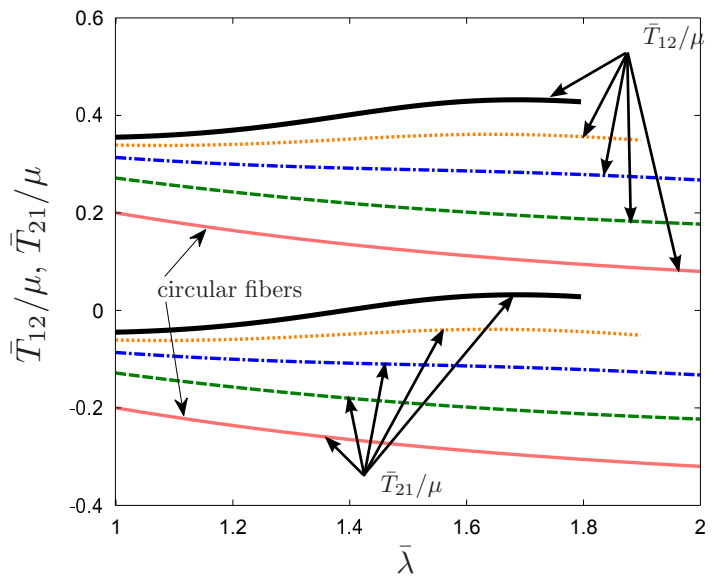

(b)

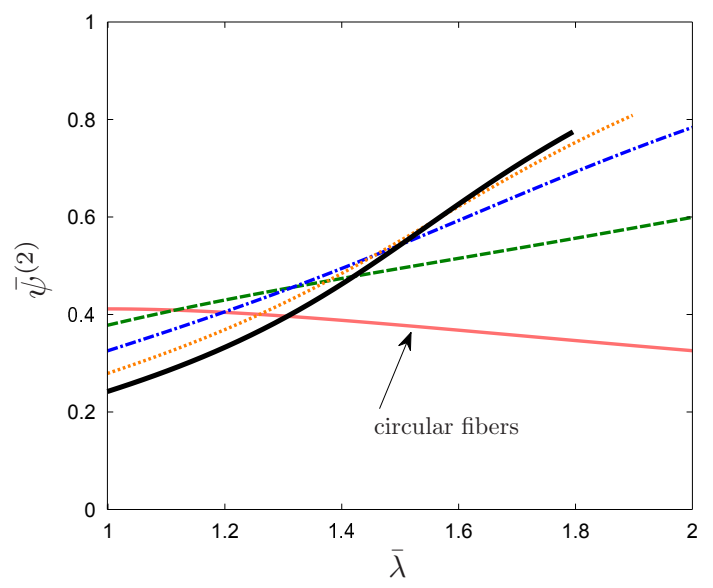

(d)

Figure 9: This figure shows the effect of the externally applied torques on the stress-stretch response of the composite under axis-aligned pure shear deformations of the form (96), as obtained by (a) shows $\bar{T}_{11}-\bar{T}_{22}$, (b) $\bar{T}_{12}$ and $\bar{T}_{21}$, (c) $\bar{T}_{12}-\bar{T}_{21}$, and (d) $\bar{\psi}^{(2)}$. In this figure $c_{0}=0.2, l_{3}=2 \mu, J_{m}=\infty$, and $\bar{\theta}=0$.

reduces to the homogenization model of Lopez-Pamies and Ponte Castañeda (2006b) when the "extra" torque on the fibers vanishes, and thus provides a generalization of the earlier model to account for the additional fiber torques, or alternatively, for prescribed fiber rotations. In addition, it is important to emphasize that the corresponding linearized results, including expression (84) for the torque-rotation relation, as well as expressions (85) and (86) for the effective stored-energy functions under prescribed torques and rotations, respectively, are also new, since they provide generalizations of the earlier results of Walpole (1991) for the effect of a torque on a single ellipsoidal particle - to account for non-dilute particle concentrations.

While the homogenization method developed in this work to deal with the effects of externally prescribed torques and rotations has been applied for special case of aligned cylindrical fibers subjected to in-plane fiber rotations and macroscopic deformations, the basic approach can be used for general ellipsoidal parti- 
cles subjected to general loading conditions (arbitrarily oriented torques and general deformations on the boundary). As such, it could be used in particular to account for externally applied torques, or rotations, on random distributions of aligned ellipsoidal particles embedded in an elastomer phase, building on the recent work of Avazmohammadi and Ponte Castañeda (2014a,b) for such soft-matrix composite materials. The methodology developed in this work could also be used in the context of other homogenization approaches, such as the one developed recently by deBotton (2005) and Lopez-Pamies and Idiart (2010) for sequentially laminated microstructures. Finally, it is remarked that the results of this work for the macroscopic response of fiber-reinforced composites with prescribed in-plane rotations can be readily used to account for magnetic (electric) torques in MAEs (DECs) by means of the "partial decoupling strategy" of Ponte Castañeda and Siboni (2012). Such an approach would be expected to improve the accuracy of currently available estimates in which the extra rotations due to the electrostatic (or magnetostatic) torques are neglected (e.g., see Galipeau and Ponte Castañeda, 2013; Siboni and Ponte Castañeda, 2014, in the context of MAEs and DECs, respectively).

To check the validity of our model, we have made comparisons with previously available results for a single fiber embedded in an elastic matrix both in the context of infinitesimal and finite deformations. In particular, we compared expression (74) for the relation between the applied body couple and the (average) in-plane rotation of the fibers in a composite with a finite volume fraction of fibers with the results of Walpole (1991) for a single fiber of elliptical cross section undergoing infinitesimal rotation, and it was found that the new result agrees exactly with the earlier result in the limit as the volume fraction of the fibers tends to zero and the imposed macroscopic strain is small. In addition, the new results were also compared with the exact analytical solutions of Rivlin (1949) and Kanner and Horgan (2008) for cylindrical shells undergoing finite azimuthal shear. In this case, the new results were found to be consistent only to second order in the rotation angle for neo-Hookean materials. However, it was found that the agreement was fairly good for rotation angles of up to $\pi / 4$. While such angles do not correspond to very large rotations, they are sufficiently large to account for the additional magnetically or electrically induced rotations in MAEs and DECs (Galipeau and Ponte Castañeda, 2013; Siboni and Ponte Castañeda, 2014), at least when the elastomeric matrix is not too soft, so that the particles can transmit sufficiently large field-activated strains. In particular, it will be of interest to explore the effect of such additional field-induced rotations on the onset of possible instabilities in MAEs and DECs (Agoras et al., 2009; Michel et al., 2010; Galipeau and Ponte Castañeda, 2013; Siboni et al., 2015). At this point, it should also be emphasized that expression (74) for the finite rotation of a random distribution of aligned fibers in an elastomeric matrix accounts for the finite values of the volume fractions of the fibers, general elliptical shapes for their cross-section, as well as for externally applied finite strains, and is therefore much more general than the previously available results. In addition, the work provides expressions for the effect of the particle rotations on the macroscopic stress-stretch relation of the composite by means of the estimate (81) for the homogenized stored-energy function of the composite subject to prescribed fiber rotations. 
As already mentioned, the errors in the predictions of the model for the torque-rotation relation at large rotations can be at least partially attributed to certain simplifying assumptions in the selection of the moduli of the LCC used to estimate the properties of the finite-elastic composites. In fact, we have made use of the same form for the moduli that was used by Lopez-Pamies and Ponte Castañeda (2006b) in the earlier solution for the case where only the macroscopic deformation is imposed on the material (i.e., no additional particle torques). While these simplifying assumptions have allowed us to take advantage of the earlier asymptotic analysis carried out by Lopez-Pamies and Ponte Castañeda (2006b) to handle the incompressibility limit and thereby obtain a simple analytical form, it is expected that the presence of additional torques would break the symmetry of the moduli used for the case of no additional torques. For this reason, it is expected that the use of more general forms for the moduli in the LCC should lead to improved estimates for the torquerotation relation of the fiber-reinforced elastomers, albeit at the expense of a somewhat more challenging asymptotic analysis. We expect to address this issue in future work.

We have also investigated in some detail the effect of the microstructure and macroscopic deformation on the torque-rotation response of fibers. Our results show that increasing the concentration or aspect ratio of the fibers results in stiffer torque-rotation responses, as expected. To elucidate the effects of macroscopic loading, we considered both axis-aligned pure shear and simple shear deformations. In particular, for pure shears that result in stretching the composite in the direction perpendicular to the longer in-plane axis of the fibers, we observe a significant softening effect in the torque-rotation response. We also observe that as the stretch (perpendicular to the longer axis of the fibers) increases the initial slope of the torque-rotation response curve tends to zero, suggesting the possible development of instabilities. On the other hand, when the sample is stretched in the direction parallel to the long axis of the fibers, a stiffening effect is observed. For simple shear deformation of the sample, our results predict non-zero rotations for the fibers, even in the absence of externally applied body couples. Such rotations are the consequence of the macroscopic pure shear and change according to the direction and magnitude of the applied torque.

Finally, we explored the effect of externally applied torques on the overall stress-stretch response of fiberreinforced composites. Our results for the special case when the pure shear deformation is aligned with the fiber axis show that the externally applied torques have a weak effect on the the normal components of the macroscopic stress, while they have a more noticeable effect on the shear components. Consistently with the general formulation for homogeneous materials in the presence of external body couples, our results show that the effective Cauchy stress for the composite is no longer symmetric in the presence of externally applied torques on the fibers. In fact, our results confirm that the anti-symmetric part of the macroscopic Cauchy stress is equal to the applied macroscopic (or net) torque per unit volume of the composite specimen.

\section{Acknowledgments}

This material is based upon work supported by the National Science Foundation, which began under Grant No. CMMI-1068769 and was completed under Grant No. DMS-1108847. 


\section{Appendix A. The connection between the eigenstresses and externally applied body couples}

In this appendix we show that a uniform eigenstress $\boldsymbol{M}$ that is applied on a hyperelastic material with objective stored energy $W(\boldsymbol{F})$ can be reinterpreted as an externally applied body couple. With this goal in mind, we note that in the presence of the eigenstress $\boldsymbol{M}$ the (first) Piola-Kirchhoff and Cauchy stresses in the hyperelastic phase may be written as

$$
\boldsymbol{S}=\frac{\partial W}{\partial \boldsymbol{F}}+\boldsymbol{M} \quad \text { and } \quad \boldsymbol{T}=J^{-1} \frac{\partial W}{\partial \boldsymbol{F}} \boldsymbol{F}^{T}+\boldsymbol{m},
$$

where $\boldsymbol{m}:=J^{-1} \boldsymbol{M} \boldsymbol{F}^{T}$ is the Eulerian counterpart of $\boldsymbol{M}$. Next we make use of the integral form for the (quasi-static) conservation of angular momentum equation for an arbitrary body part, as given by

$$
\int_{\partial \mathcal{B}_{0}} \mathbf{x} \times(\boldsymbol{S N}) d S+\int_{\mathcal{B}_{0}} \rho^{0} \mathbf{c}^{0}(\mathbf{X}) d V=0, \quad \text { and } \quad \int_{\partial \mathcal{B}} \mathbf{x} \times(\boldsymbol{T} \mathbf{n}) d s+\int_{\mathcal{B}} \rho \mathbf{c}(\mathbf{x}) d v=0,
$$

in the reference and current configurations, respectively. Here, $\mathcal{B}_{0}$ denotes the arbitrary body part in the reference (undeformed) configuration with unit outward normal $\mathbf{N}$, while $\mathcal{B}$ denotes the corresponding body part in the current (deformed) configuration with unit outward normal $\mathbf{n}$. Substituting the stresses $\boldsymbol{S}$ and $\boldsymbol{T}$ from (A.1) into the conservation of angular momentum equations (A.2), and using appropriate forms of the divergence theorem and the fact that the domain of integration in the integrals is arbitrary, it follows that

$$
\boldsymbol{F} \boldsymbol{M}^{T}-\boldsymbol{M} \boldsymbol{F}^{T}=2 \rho^{0} \boldsymbol{C}^{0}(\mathbf{X}), \quad \text { or } \quad \boldsymbol{m}-\boldsymbol{m}^{T}=2 \rho \boldsymbol{C}(\mathbf{x}) .
$$

In these expressions

$$
C_{m n}^{0}(\mathbf{X}):=\frac{1}{2} \varepsilon_{m n i} c_{i}^{0}(\mathbf{X}) \quad \text { and } \quad C_{m n}(\mathbf{x}):=\frac{1}{2} \varepsilon_{m n i} c_{i}(\mathbf{x}),
$$

are, respectively, anti-symmetric second-order tensors corresponding to the vector of body couple in the

reference configuration, $\mathbf{c}^{0}(\mathbf{X})$, and in the deformed configuration, $\mathbf{c}(\mathbf{x})$. Note that in obtaining (A.3) we used the objectivity of the stored-energy function $W(\boldsymbol{F})$ to make the identity

$$
\frac{\partial W}{\partial \boldsymbol{F}} \boldsymbol{F}^{T}-\boldsymbol{F}\left(\frac{\partial W}{\partial \boldsymbol{F}}\right)^{T}=0
$$

Note that the eigenstress $\boldsymbol{M}$, when written in the Eulerian form, corresponds to the anti-symmetric part of the Cauchy stress and therefore to the body couple.

It is emphasized that, unlike $\boldsymbol{M}$, which is taken to be uniform here, the local body couple $\boldsymbol{C}^{0}(\mathbf{X})$ will not in general be uniform, due to the potential dependence of the local deformation gradient $\boldsymbol{F}$ on $\mathbf{X}$. However, as discussed in the text for the special cases of two-phase composites where the homogenization estimates predict uniform deformation fields in the inclusion phase (i.e., $\boldsymbol{F}(\mathbf{X})=\overline{\boldsymbol{F}}^{(2)}$ for $\mathbf{X} \in \Omega_{0}^{(2)}$ ), the body couple corresponding to the uniform eigenstress in the inclusion phase turns out to be uniform.

\section{Appendix B. Levin relations for two-phase linear thermoelastic composites}

In this appendix we provide explicit expressions for the effective stored energy of linear two-phase composites with particulate microstructures. Thus, consider a two-phase composite consisting of linear phases 
with the following quadratic energies

$$
W_{L C C}^{(r)}=f^{(r)}+\boldsymbol{T}^{(r)} \cdot \boldsymbol{F}+\frac{1}{2} \boldsymbol{F} \cdot \mathbb{L}^{(r)} \boldsymbol{F}, \quad \text { for } \quad r=1,2 .
$$

At this stage, $f^{(r)}, \boldsymbol{T}^{(r)}$, and $\mathbb{L}^{(r)}$ are given quantities which may be replaced by the corresponding coefficients obtained from the linearization of the nonlinear phase energies. Using a generalization of the Levin relations for two-phase (i.e., $r=1,2$ ) thermoelastic composites (Levin, 1967), it is found that

$$
\hat{W}_{L C C}=\tilde{f}+\tilde{\boldsymbol{T}} \cdot \overline{\boldsymbol{F}}+\frac{1}{2} \overline{\boldsymbol{F}} \cdot \tilde{\mathbb{L}} \overline{\boldsymbol{F}}
$$

where

$$
\begin{aligned}
\tilde{f} & :=\bar{f}+\frac{1}{2} \Delta \boldsymbol{T} \cdot\left[(\Delta \mathbb{L})^{-1}(\tilde{\mathbb{L}}-\overline{\mathbb{L}})(\Delta \mathbb{L})^{-1}\right] \Delta \boldsymbol{T} \\
\tilde{\boldsymbol{T}} & :=\overline{\boldsymbol{T}}+(\tilde{\mathbb{L}}-\overline{\mathbb{L}})(\Delta \mathbb{L})^{-1} \Delta \boldsymbol{T} .
\end{aligned}
$$

Here $\Delta \boldsymbol{T}:=\boldsymbol{T}^{(1)}-\boldsymbol{T}^{(2)}, \Delta \mathbb{L}:=\mathbb{L}^{(1)}-\mathbb{L}^{(2)}$, and $\bar{f}, \overline{\boldsymbol{T}}$ and $\overline{\mathbb{L}}$ are the volume averages of the corresponding local variables. For particulate microstructures with ellipsoidal symmetry (Ponte Castañeda and Willis, 1995), we make use of Hashin-Shtrikman type estimates for the effective modulus. Thus, $\tilde{\mathbb{L}}$ will be taken to be

$$
\tilde{\mathbb{L}}=\mathbb{L}^{(1)}+c_{0}\left[\left(1-c_{0}\right) \mathbb{P}-(\Delta \mathbb{L})^{-1}\right]^{-1},
$$

where $c_{0}$ is the volume fraction of the particle phase and $\mathbb{P}$ is the usual microstructural Eshelby-type tensor (with major symmetries but not necessarily minor symmetries). The microstructural tensor $\mathbb{P}$ for composites consisting of inclusions with general ellipsoidal shapes, as characterized by the second-order shape tensor $\boldsymbol{Z}_{0}$, may be expressed as

$$
\mathbb{P}:=\frac{\operatorname{det} \boldsymbol{Z}_{0}}{4 \pi} \int_{|\boldsymbol{\xi}|=1} \mathbb{H}(\boldsymbol{\xi})\left|\boldsymbol{Z}_{0}^{T} \boldsymbol{\xi}\right|^{-3} d S
$$

where the fourth-order tensor $\mathbb{H}$ is defined in terms of the acoustic tensor $K_{i k}=L_{i j k l}^{(1)} \xi_{j} \xi_{l}$ via

$$
H_{i j k l}(\boldsymbol{\xi}):=K_{i k}^{-1} \xi_{j} \xi_{l}
$$

Note that the second-order tensor $\boldsymbol{Z}_{0}$ describes the ellipsoidal shape of the inclusions, i.e.,

$$
\mathcal{I}_{0}:=\left\{\mathbf{X} \mid \mathbf{X} \cdot \boldsymbol{Z}_{0}^{-2} \mathbf{X} \leq 1\right\}
$$

For composites consisting of long cylindrical fibers with elliptical shapes for the cross-section the above expression for the $\mathbb{P}$-tensor simplifies to

$$
\mathbb{P}:=\frac{\operatorname{det} \boldsymbol{Z}_{0}}{2 \pi} \int_{\xi_{1}^{2}+\xi_{2}^{2}=1} \mathbb{H}\left(\xi_{1}, \xi_{2}, \xi_{3}=0\right)\left|\boldsymbol{Z}_{0}^{T} \boldsymbol{\xi}\right|^{-1} d S
$$

Having the expression for the effective response of the above composite we can use the identity

$$
\overline{\boldsymbol{F}}_{L C C}^{(2)}=\frac{1}{c_{0}} \frac{\partial \hat{W}_{L C C}}{\partial \boldsymbol{T}^{(2)}}
$$


to obtain an expression for the average deformation gradient in the inclusion phase. Towards this end, we first note that

$$
\frac{\partial}{\partial \boldsymbol{T}^{(2)}} \tilde{f}:=-\mathbb{Y} \Delta \boldsymbol{T} \text { and } \frac{\partial}{\partial \boldsymbol{T}^{(2)}} \tilde{\boldsymbol{T}} \cdot \overline{\boldsymbol{F}}:=c_{0} \overline{\boldsymbol{F}}-\mathbb{Y} \Delta \mathbb{L} \overline{\boldsymbol{F}}
$$

where

$$
\mathbb{Y}:=(\Delta \mathbb{L})^{-1}(\tilde{\mathbb{L}}-\overline{\mathbb{L}})(\Delta \mathbb{L})^{-1}
$$

Then taking the derivative of (B.2) with respect to $\boldsymbol{T}^{(2)}$, and substituting identities (B.10) into the resulting expression, we obtain

$$
\begin{aligned}
\overline{\boldsymbol{F}}-\overline{\boldsymbol{F}}_{L C C}^{(2)} & =\frac{\mathbb{Y}}{c_{0}}[\Delta \boldsymbol{T}+\Delta \mathbb{L} \overline{\boldsymbol{F}}] \\
& =\frac{\mathbb{Y}}{c_{0}}\left[\Delta \boldsymbol{T}+\Delta \mathbb{L} \overline{\boldsymbol{F}}_{L C C}^{(2)}+\Delta \mathbb{L}\left(\overline{\boldsymbol{F}}-\overline{\boldsymbol{F}}_{L C C}^{(2)}\right)\right] .
\end{aligned}
$$

Solving the above equation for $\overline{\boldsymbol{F}}-\overline{\boldsymbol{F}}_{L C C}^{(2)}$, we get

$$
\left\{\mathbb{I}-\frac{\mathbb{Y} \Delta \mathbb{L}}{c_{0}}\right\}\left(\overline{\boldsymbol{F}}-\overline{\boldsymbol{F}}_{L C C}^{(2)}\right)=\frac{\mathbb{Y}}{c_{0}}\left[\Delta \boldsymbol{T}+\Delta \mathbb{L} \overline{\boldsymbol{F}}_{L C C}^{(2)}\right]
$$

or

$$
\left(\overline{\boldsymbol{F}}-\overline{\boldsymbol{F}}_{L C C}^{(2)}\right)=\left\{\mathbb{I}-\frac{\mathbb{Y} \Delta \mathbb{L}}{c_{0}}\right\}^{-1} \frac{\mathbb{Y}}{c_{0}}\left[\Delta \boldsymbol{T}+\Delta \mathbb{L} \overline{\boldsymbol{F}}_{L C C}^{(2)}\right] .
$$

Given the expression (B.4) and the definition (B.11), we can show that

$$
\frac{1}{c_{0}} \mathbb{Y}=\left[\mathbb{I}-\left(1-c_{0}\right)^{-1}(\Delta \mathbb{L})^{-1} \mathbb{P}^{-1}\right]^{-1}(\Delta \mathbb{L})^{-1}
$$

and

$$
\left\{\mathbb{I}-\frac{\mathbb{Y} \Delta \mathbb{L}}{c_{0}}\right\}^{-1}=-\left(1-c_{0}\right) \mathbb{P} \Delta \mathbb{L}\left[\mathbb{I}-\left(1-c_{0}\right)^{-1}(\Delta \mathbb{L})^{-1} \mathbb{P}^{-1}\right] .
$$

Substituting (B.15) and (B.16) into (B.14), we obtain the result

$$
\overline{\boldsymbol{F}}-\overline{\boldsymbol{F}}_{L C C}^{(2)}=-\left(1-c_{0}\right) \mathbb{P}\left[\Delta \boldsymbol{T}+\Delta \mathbb{L} \overline{\boldsymbol{F}}_{L C C}^{(2)}\right] .
$$

It can also be shown that

$$
C_{L C C}^{(2)}=0,
$$

which is the direct consequence of the fact that we are using Hashin-Shtrikman estimate (B.4) for $\tilde{\mathbb{L}}$. This implies that in the inclusion phase of the LCC the deformation gradient is uniform, such that

$$
\boldsymbol{F}(\mathbf{X})=\overline{\boldsymbol{F}}_{L C C}^{(2)} \text { for } \quad \mathbf{X} \in \Omega_{0}^{(2)}
$$

\section{References}

Agoras, M., Lopez-Pamies, O., Ponte Castañeda, P., 2009. Onset of macroscopic instabilities in fiberreinforced elastomers at finite strain. Journal of the Mechanics and Physics of Solids 57, 1828 - 1850. doi:http://dx.doi.org/10.1016/j.jmps.2009.08.001. 
Avazmohammadi, R., Ponte Castañeda, P., 2014a. On the macroscopic response, microstructure evolution, and macroscopic stability of short-fibre-reinforced elastomers at finite strains: I- analytical results. Philosophical Magazine 94, 1031-1067. doi:10.1080/14786435.2013.878048.

Avazmohammadi, R., Ponte Castañeda, P., 2014b. On the macroscopic response, microstructure evolution, and macroscopic stability of short-fiber-reinforced elastomers at finite strains: II- representative examples. Philosophical Magazine 94, 1068-1094. doi:10.1080/14786435.2013.878049.

Avazmohammadi, R., Ponte Castañeda, P., 2015. Macroscopic constitutive relations for elastomers reinforced with short aligned fibers: instabilities and post-bifurcation response. Journal of the Mechanics and Physics of Solids, in press. doi:10.1016/j.jmps.2015.07.007.

deBotton, G., 2005. Transversely isotropic sequentially laminated composites in finite elasticity. Journal of the Mechanics and Physics of Solids 53, 1334 - 1361. doi:10.1016/j.jmps.2005.01.006.

Eshelby, J.D., 1957. The Determination of the Elastic Field of an Ellipsoidal Inclusion, and Related Problems. Proc. R. Soc. Lond. A 241, 376-396. doi:10.1098/rspa.1957.0133.

Galipeau, E., Ponte Castañeda, P., 2013. A finite-strain constitutive model for magnetorheological elastomers: Magnetic torques and fiber rotations. Journal of the Mechanics and Physics of Solids 61, 10651090. doi:10.1016/j.jmps.2012.11.007.

Gent, A.N., 1996. A New Constitutive Relation for Rubber. Rubber Chemistry and Technology 69, 59-61. doi:10.5254/1.3538357.

Hill, R., 1972. On Constitutive Macro-Variables for Heterogeneous Solids at Finite Strain. Proceedings of the Royal Society of London. A. Mathematical and Physical Sciences 326, 131-147. doi:10.1098/rspa.1972.0001.

Idiart, M.I., Ponte Castañeda, P., 2007. Field statistics in nonlinear composites: I. theory. Proceedings of the Royal Society A: Mathematical, Physical and Engineering Science 463, 183-202. doi:10.1098/rspa.2006.1756.

Kanner, L.M., Horgan, C.O., 2008. Inhomogeneous shearing of strain-stiffening rubber-like hollow circular cylinders. International Journal of Solids and Structures 45, 5464-5482. doi:10.1016/j.ijsolstr.2008.05.030.

Levin, V.M., 1967. Thermal expansion coefficients of heterogeneous materials. Mekh. Tverd. Tela. 2, 83-94.

Lopez-Pamies, O., Idiart, M.I., 2010. Fiber-reinforced hyperelastic solids: a realizable homogenization constitutive theory. Journal of Engineering Mathematics 68, 57-83. doi:10.1007/s10665-009-9359-y.

Lopez-Pamies, O., Ponte Castañeda, P., 2006a. On the overall behavior, microstructure evolution, and macroscopic stability in reinforced rubbers at large deformations: I-theory. Journal of the Mechanics and Physics of Solids 54, 807-830. doi:10.1016/j.jmps.2005.10.006. 
Lopez-Pamies, O., Ponte Castañeda, P., 2006b. On the overall behavior, microstructure evolution, and macroscopic stability in reinforced rubbers at large deformations: II-Application to cylindrical fibers. Journal of the Mechanics and Physics of Solids 54, 831-863. doi:10.1016/j.jmps.2005.10.010.

Michel, J.C., Lopez-Pamies, O., Ponte Castañeda, P., Triantafyllidis, N., 2010. Microscopic and macroscopic instabilities in finitely strained fiber-reinforced elastomers. Journal of the Mechanics and Physics of Solids 58, 1776-1803. doi:10.1016/j.jmps.2010.08.006.

Ponte Castañeda, P., 2002. Second-order homogenization estimates for nonlinear composites incorporating field fluctuations: I-theory. Journal of the Mechanics and Physics of Solids 50, 737-757. doi:10.1016/S00225096(01)00099-0.

Ponte Castañeda, P., Galipeau, E., 2011. Homogenization-based constitutive models for magnetorheological elastomers at finite strain. J. Mech. Phys. Solids 59, 194 - 215. doi:10.1016/j.jmps.2010.11.004.

Ponte Castañeda, P., Siboni, M.H., 2012. A finite-strain constitutive theory for electro-active polymer composites via homogenization. Int. J. Non-Linear Mech. 47, 293-306. doi:10.1016/j.ijnonlinmec.2011.06.012.

Ponte Castañeda, P., Willis, J.R., 1995. The effect of spatial distribution on the effective behavior of composite materials and cracked media. J. Mech. Phys. Solids 43, 1919-1951. doi:10.1016/0022-5096(95)00058-Q.

Rivlin, R.S., 1949. Large elastic deformations of isotropic materials. vi. further results in the theory of torsion, shear and flexure. Philosophical Transactions of the Royal Society of London. Series A, Mathematical and Physical Sciences 242, 173-195.

Siboni, M.H., Avazmohammadi, R., Ponte Castañeda, P., 2015. Electromechanical instabilities in fiberconstrained, dielectric-elastomer composites subjected to all-around dead-loading. Mathematics and Mechanics of Solids 20, 729-759. doi:10.1177/1081286514551501.

Siboni, M.H., Ponte Castañeda, P., 2012. A magnetically anisotropic, ellipsoidal inclusion subjected to a non-aligned magnetic field in an elastic medium. C. R. Mécanique 340, $205-218$. doi:10.1016/j.crme.2012.02.003.

Siboni, M.H., Ponte Castañeda, P., 2014. Fiber-constrained, dielectric-elastomer composites: finitestrain response and stability analysis. Journal of the Mechanics and Physics of Solids 68, 211-238. doi:10.1016/j.jmps.2014.03.008.

Walpole, L.J., 1991. A rotated rigid ellipsoidal inclusion in an elastic medium. Proceedings of the Royal Society of London. Series A: Mathematical and Physical Sciences 433, 179-207. doi:10.1098/rspa.1991.0042.

Willis, J.R., 1977. Bounds and self-consistent estimates for the overall properties of anisotropic composites. Journal of the Mechanics and Physics of Solids 25, 185-202. doi:10.1016/0022-5096(77)90022-9. 